\title{
Antiproton Production and Antideuteron Production Limits in Relativistic Heavy Ion Collisions
}

\author{
T.A. Armstrong, ${ }^{(7, *)}$ K.N. Barish, ${ }^{(3)}$ S. Batsoulli, ${ }^{(12)}$ S.J. Bennett, ${ }^{(11)}$ A. Chikanian, ${ }^{(12)}$ \\ S.D. Coe,${ }^{(12, \dagger)}$ T.M. Cormier, ${ }^{(11)}$ R. Davies, ${ }^{(8, \ddagger)}$ C.B. Dover, ${ }^{(1, \S)}$ P. Fachini, ${ }^{(11)}$ B. Fadem, ${ }^{(4)}$ \\ L.E. Finch, ${ }^{(12)}$ N.K. George, ${ }^{(12)}$ S.V. Greene, ${ }^{(10)}$ P. Haridas, ${ }^{(6)}$ J.C. Hill, ${ }^{(4)}$ A.S. Hirsch, ${ }^{(8)}$ \\ R. Hoversten, ${ }^{(4)}$ H.Z. Huang, ${ }^{(2)}$ B.S. Kumar, ${ }^{(12, \|)}$ T. Lainis, ${ }^{(9)}$ J.G. Lajoie, ${ }^{(4)}$ Q. Li, ${ }^{(11)}$ \\ B. Libby, ${ }^{(4, \boldsymbol{\Upsilon} \uparrow)}$ R.D. Majka, ${ }^{(12)}$ T.E. Miller, ${ }^{(10)}$ M.G. Munhoz, ${ }^{(11)}$ J.L. Nagle, ${ }^{(12, * *)}$ \\ I.A. Pless, ${ }^{(6)}$ J.K. Pope, ${ }^{(12, \dagger \dagger)}$ N.T. Porile, ${ }^{(8)}$ C.A. Pruneau, ${ }^{(11)}$ M.S.Z. Rabin, ${ }^{(5)}$ \\ J.D. Reid, ${ }^{(10)}$ A. Rimai, ${ }^{(8, \sharp \ddagger)}$ A. Rose,${ }^{(10)}$ F.S. Rotondo, ${ }^{(12, \S \S)}$ J. Sandweiss, ${ }^{(12)}$ \\ R.P. Scharenberg, ${ }^{(8)}$ A.J. Slaughter, ${ }^{(12)}$ G.A. Smith, ${ }^{(7)}$ M.L. Tincknell, ${ }^{(8,\|\|)}$ \\ W.S. Toothacker, ${ }^{(7)}$ G. Van Buren, ${ }^{(6)}$ F.K. Wohn, ${ }^{(4)}$ Z. Xu, ${ }^{(12)}$
} (The E864 Collaboration)

(1) Brookhaven National Laboratory, Upton, New York 11973

(2) University of California at Los Angeles, Los Angeles, California 90095

(3) University of California at Riverside, Riverside, California 92521

(4) Iowa State University, Ames, Iowa 50011

(5) University of Massachusetts, Amherst, Massachusetts 01003

(6) Massachusetts Institute of Technology, Cambridge, Massachusetts 02139

(7) Pennsylvania State University, University Park, Pennsylvania 16802

(8) Purdue University, West Lafayette, Indiana 47907

(9) United States Military Academy, West Point

(10) Vanderbilt University, Nashville, Tennessee 37235

(11) Wayne State University, Detroit, Michigan 48201

(12) Yale University, New Haven, Connecticut 06520 


\begin{abstract}
We present results from Experiment 864 for antiproton production and antideuteron limits in $\mathrm{Au}+\mathrm{Pb}$ collisions at $11.5 \mathrm{GeV} / \mathrm{c}$ per nucleon. We have measured invariant multiplicities for antiprotons for rapidities $1.4<y<2.4$ at low transverse momentum as a function of collision geometry. When compared with the results from Experiment 878 our measurements suggest a significant contribution to the measured antiproton yield from the decay of strange antibaryons. We have also searched for antideuterons and see no statistically significant signal. Thus, we set upper limits on the production at approximately $3 \times 10^{-7}$ per $10 \%$ highest multiplicity $\mathrm{Au}+\mathrm{Pb}$ interaction.
\end{abstract}

PACS numbers: $25.75 .+\mathrm{r}$ 


\section{INTRODUCTION}

Antimatter production in relativistic heavy ion collisions has been proposed as an excellent probe of the collision dynamics and possible phase transition to a quark-gluon plasma [1]. The production of antiprotons at AGS energies $(10-15 \mathrm{GeV} / \mathrm{c})$ is near threshold in nucleon-nucleon collisions. Therefore multiple collisions, resonance excitations, and collective effects may play a major role in significantly increasing the overall production rates [2]. Strange antibaryons, due to their larger mass and additional antistrange quark, are even further suppressed in initial nucleon-nucleon collisions. However, strangeness saturation and antimatter enhancement have long been predictions of quark-gluon plasma states. Thus, understanding the yields of non-strange and strange antibaryons is an important tool for distinguishing between various sources of enhanced production.

Antibaryons have a large annihilation cross section (particularly at low relative momentum), reaching levels of hundreds of millibarns. Thus, in these baryon rich colliding systems, there can be significant annihilation losses before they escape the collision region. The final experimentally measured yields represent the initial production minus the annihilation losses. The annihilation process in free space is well understood and experimentally parametrized; however, this annihilation may be modified in the dense particle environment where the initial attraction of baryon and antibaryon may be disturbed [3].

Antideuteron production at AGS energies is actually below the energy threshold in single nucleon-nucleon collisions and thus antideuterons are expected to only be created through the coalescence of separately produced antiprotons and antineutrons which are close enough together in coordinate space and phase space at freeze-out. Because of the large energy required for their production, antideuteron yields are an excellent measure of the system's thermal temperature (assuming antimatter is equilibrated in these collisions). Also, coa-

lescence yields would yield information about the spatial distribution of antinucleons (as a form of two particle correlation) [4].

In the next section we describe the E864 spectrometer and the antiproton data sets. In 
section three we present the measured antiproton invariant multiplicities and compare them with measurements made by other experiments. These comparisons lead us to consider the possibility of enhanced production of strange antibaryons in these collisions. Antimatter correlations in the form of events with two antiprotons and antideuteron production are discussed in section four.

\section{EXPERIMENT 864}

\section{A. The E864 Spectrometer}

Experiment 864 was designed to search for novel forms of matter (particularly strange quark matter, or "strangelets") produced in heavy ion collisions at the Brookhaven AGS facility [5]. In order to conduct this search, E864 has a large geometric acceptance and operates at a high data rate. A diagram of the spectrometer is shown in Figure 1. Secondary particles produced from the $\mathrm{Au}+\mathrm{Pb}$ reaction which are within the geometric acceptance traverse two dipole magnets (M1 and M2) and then multiple downstream tracking stations. Non-interacting beam particles and beam fragments near zero degrees pass above the downstream spectrometer in a vacuum chamber, thus reducing interactions which would otherwise produce background hits in the detectors. The experiment does not measure at zero degrees (zero transverse momentum), but particles with at least 15 milliradians of angle pass through an exit window in the vacuum chamber.

Charged particles are tracked using three time-of-flight (TOF) hodoscopes and two straw tube stations. The hodoscopes yield three independent charge measurements of $\mathrm{dE} / \mathrm{dx}$ over the $1 \mathrm{~cm}$ thickness of the scintillator slats and provide three space-time points with time resolutions on the order of $120-150$ ps. The straw stations provide more accurate position information for track projection back into the magnetic field region. Particles are identified by their time-of-flight from the target (yielding the velocity) and momentum. The momentum is determined by combining the charge measurement with the rigidity ( $\mathrm{R}$ 
$=\mathrm{p} / \mathrm{Z})$ from the track projection in the bend plane into the field region. The redundant measurements allow for excellent background rejection of ghost tracks and tracks originating from downstream interactions.

A second particle identification measurement can be made using a hadronic calorimeter located at the end of the spectrometer [6]. The calorimeter consists of 754 individual towers constructed from lead sheets with scintillating fibers running almost parallel to the incident particle trajectory. The calorimeter yields good timing information $(\sigma \approx 400 \mathrm{ps}$ for hadrons) and excellent hadronic energy resolution of $3.5 \%+34 \% / \sqrt{E}$ (with $\mathrm{E}$ in $\mathrm{GeV}$ ). For baryons, the calorimeter measures the particle's kinetic energy, which when combined with timeof-flight information gives a measure of the particle mass. For antibaryons, the energy measurement also includes the annihilation energy of the antibaryon and its annihilation partner.

The experiment is able to perform high sensitivity searches by running at high rate with a special "late energy" trigger (LET) [7]. The time and energy signals from each of 616 fiducial calorimeter towers are digitized in flash ADC's and TDC's and used as inputs to a lookup table, which is programmed to select the particles of interest. Because there are many slow neutrons and many fast high energy protons, a simple time cut or an energy cut was determined to be insufficient for the trigger. The late energy trigger allows for the rejection of both of these abundant particles, while effectively triggering on slow (mid-rapidity) particles which deposit a large amount of energy. An antiproton of the same momentum as a proton or neutron will deposit an additional annihilation energy. If the $\mathrm{Au}+\mathrm{Pb}$ interaction yields no towers firing the trigger, then a fast clear is sent out to the digitizers and the data is not recorded. The trigger yields an enhancement factor for antiprotons, antideuterons and strangelets of approximately 50 (under running conditions appropriate for each species).

In order to determine the collision geometry (impact parameter) a charged particle multiplicity counter is used. The E864 multiplicity counter [8] is an annular piece of fast BC420 scintillator placed around the beam pipe $13 \mathrm{~cm}$ downstream of the target and tipped at an angle of $8^{\circ}$ to the vertical. It is $1 \mathrm{~cm}$ thick and subtends the angular range of $16.6^{\circ}$ 
to $45.0^{\circ}$. The annulus is separated into four quadrants and each quadrant is viewed by a photomultiplier tube. The total signal measured with this counter is proportional to the charged particle multiplicity of the collision. The integrated signal from the sum of the four quadrants is used to trigger on the centrality of the events by selecting events with a signal larger than a given threshold.

\section{B. Data Sets}

The data used in this analysis was collected in two separate data taking periods. During the fall of 1995, the late energy trigger was strobed on the $10 \%$ most central $\mathrm{Au}+\mathrm{Pb}$ interactions with the spectrometer magnets set for optimal acceptance for antideuterons (referred to as the "-0.75T" field setting). The LET curve was set to yield an enhancement factor of $\sim 50$ for antideuterons and negative strangelets. The data set includes over 90 million recorded events, which effectively sampled approximately six billion central interactions. From this sample, over 50,000 antiprotons were identified. The mass distributions of antiprotons from a single rapidity and transverse momentum bin are shown in Figure 2 .

In the fall 1996 run, the LET was strobed on minimum-bias (93\% of the geometric cross section) $\mathrm{Au}+\mathrm{Pb}$ interactions and the LET curve and the spectrometer magnets were set for optimal antiproton acceptance (referred to as the "-0.45T" field setting). The LET yielded an enhancement factor for antiprotons $>50$ under these conditions. However, in order to use the trigger effectively, the region of the calorimeter dominated by neutrons from the interaction had to be excluded. This reduced the geometric acceptance by roughly a factor of two. The data sample included 45 million recorded minimum bias interactions and approximately 50,000 antiprotons. These data samples represent the largest statistics for antiprotons produced in heavy ion collisions at the BNL-AGS.

In both data sets, the beam momentum was measured using the E864 spectrometer magnets and a downstream beam counter located in the beam dump. The beam momentum of $11.5 \mathrm{GeV} / \mathrm{c}$ per nucleon was consistent with the beam momentum reported at extraction 
from the accelerator once energy losses due to material in the E864 beam line were properly accounted for. The Au beam was incident on a $30 \% \mathrm{~Pb}$ target for the 1995 data set, while a $10 \% \mathrm{~Pb}$ target was used in 1996 .

\section{ANTIPROTON INVARIANT MULTIPLICITIES}

\section{A. E864 Measurements}

In E864 we explicitly measure the yield of antiprotons per $\mathrm{Au}+\mathrm{Pb}$ interaction as a function of centrality, and thus we directly calculate the invariant multiplicities. The invariant multiplicity for antiprotons is determined as follows:

$$
\frac{1}{2 \pi \mathrm{p}_{\mathrm{t}}} \frac{\mathrm{d}^{2} \mathrm{~N}}{\mathrm{dydp_{ \textrm {T } }}}=\frac{1}{2 \pi \overline{\mathrm{p}_{\mathrm{t}}} \Delta \mathrm{y} \Delta \mathrm{p}_{\mathrm{T}}} \frac{\mathrm{N}_{\text {counts }}}{\mathrm{N}_{\text {sampled }}} \frac{1}{\epsilon_{\text {detect }} \times \epsilon_{\text {accept }} \times \epsilon_{\text {trigger }}}
$$

The total number of antiprotons $N_{\text {counts }}$ is determined in each separate bin in phase space and divided by the total number of sampled $\mathrm{Au}+\mathrm{Pb}$ interactions $N_{\text {sampled }}$. The counted antiprotons include only those antiprotons which fired the LET. Since the detector does not measure all the antiprotons produced in a given region of phase space, the invariant multiplicity must be corrected for the missed particles. These missed antiprotons are the result of the experiment's finite acceptance and various tracking and triggering efficiencies. The acceptance $\epsilon_{\text {accept }}$ and detection efficiency $\epsilon_{\text {detect }}$ are calculated using a GEANT [9] simulation of the experiment in conjunction with real data. This simulation also included losses due to antiproton annihilation in the target as part of the acceptance correction. The production of antiprotons due to reinteraction of particles from the primary interaction with target nuclei was also considered and found to be negligible.

The LET efficiency $\epsilon_{\text {trigger }}$ is determined in each kinematic bin. This efficiency is determined in one of two ways: for antiprotons measured in the 1995 ("-0.75T") $10 \%$ central data where the efficiency was somewhat low, a sample of antiprotons that did not fire the trigger was used to determine the efficiency. In the 1996 ("-0.45T") data, where the LET curve was 
set for higher efficiency $(\sim 75 \%)$, the efficiency was determined from a Monte Carlo of the calorimeter response.

The data from E864 is measured in a range of $50<p_{T}<275 \mathrm{MeV} / \mathrm{c}$ (where the limits are a function of rapidity). The invariant multiplicities measured in E864 are approximately flat over the $p_{T}$ range measured, as shown for the 1996 data in Figure 3. Over such a small range in transverse momentum, the invariant multiplicities are not expected to change significantly. If, for example, the spectra follow a Boltzmann distribution,

$$
\frac{1}{2 \pi p_{T}} \frac{d^{2} N}{d y d p_{T}} \propto m_{T} e^{-\frac{m_{T}}{T_{B}}}
$$

(where $m_{T}=\sqrt{p_{T}^{2}+m^{2}}$ ), then with a temperature parameter of $200 \mathrm{MeV}$ the invariant multiplicity at $p_{T}=0$ is only $6 \%$ higher than at $p_{T}=150 \mathrm{MeV} / \mathrm{c}$. For comparison with other experiments, in each rapidity bin all the invariant multiplicities as a function of $p_{T}$ are fit to a constant level. This level is assigned as the invariant multiplicity at $p_{T} \approx 0$, and an additional $6 \%$ systematic error is assigned due to the $p_{T}=0$ extrapolation. It should be noted that strong radial flow could affect the $p_{T}=0$ extrapolation as well. We feel that this effect should be within the estimated systematic uncertainty since the E864 data are quite flat as a function of $p_{T}$ down to $50 \mathrm{MeV} / \mathrm{c}$ at midrapidity.

The antiproton invariant multiplicities for $10 \%$ most central $\mathrm{Au}+\mathrm{Pb}$ collisions from the 1995 data are given in Table $\mathbb{1}$ [10. It should be noted that the statistical error in this data set is dominated by the contribution from the trigger efficiency (due to counting antiprotons which did not fire the trigger). The systematic error in the 1995 data (exclusive of the $6 \%$ due to the $p_{T}=0$ extrapolation) is estimated to be $15 \%$, and is dominated by the uncertainty in the correction for the LET trigger efficiency. Systematic uncertainties also arise from our knowledge of the experimental acceptance (including the effect of the collimator in the first spectrometer magnet), track quality cuts, and the loss of tracks due to overlapping hits in the hodoscopes.

For the 1996 data, the late-energy trigger was strobed on a minimum-bias sample of events selected by the multiplicity counter. The resulting multiplicity counter ADC dis- 
tribution is shown in Figure 1 . When selecting minimum bias events, it is important to consider the effect of interactions of the beam that do not occur in the target. Using special empty-target runs we have found that non-target interactions contribute less than $10 \%$ of the multiplicity distribution at low multiplicity, while the late-energy trigger further reduces this contamination to below 1\% (see Figure 1). For the 1996 data, the antiproton invariant multiplicities are determined for different regions of the minimum bias multiplicity: 100-70\% of the full distribution, 70-30\%, 30-10\% and 10\%. These regions are shown in Figure 5. It is important to note that the LET rejection is a strong function of the multiplicity, and this must be properly accounted for when calculating the normalization in each centrality bin.

The antiproton invariant multiplicities for the four multiplicity regions used in the 1996 data set are listed in Table $\llbracket$. In addition, the full minimum bias invariant multiplicity from the 1996 data set is also listed in Table ПI. The systematic error in these data points are estimated to be $10 \%$ (again, exclusive of the $6 \%$ previously described due to the $p_{T}=0$ extrapolation). As in the 1995 data, the systematic uncertainty in the 1996 data is also dominated by the uncertainty in the determination of the LET trigger efficiency. However, the size of the correction is smaller for the 1996 data due to the overall higher efficiency of the trigger setting.

Figure 6 shows the 1995 ("-0.75T") and 1996 ("-0.45T") antiproton invariant multiplicities at $p_{T}=0$ as a function of rapidity. The Gaussians shown are fits to the combined 1995 and 1996 data, and are constrained to have a mean value at midrapidity $(y=1.6)$. There is excellent agreement between the 1995 and 1996 data in the rapidity range where the two data sets overlap. The rapidity widths measured in the data for the four multiplicty widths are $0.37 \pm 0.02(100-70 \%), 0.41 \pm 0.02(70-30 \%), 0.43 \pm 0.02(30-10 \%)$ and $0.46 \pm 0.02(10 \%)$ indicating a broadening of the rapidity spectrum at higher centrality.

Also shown in Figure 6 (as open squares) are previously reported antiproton results from data taken in 1994 ("-0.45T") [11]. It should be noted that the 1994 data is about $20 \%$ higher at midrapidity than indicated by the corresponding 1995 and 1996 data. This is within the statistical and systematic error previously quoted for the 1994 data. It is important to note 
that the 1994 data was taken with an incomplete detector: two layers of S3 were missing along with a "plug" designed to reduce the occupancy in the downstream detectors due to interactions of beam-rapidity fragments with the vacuum chamber. The presence of the plug dramatically reduced the detector occupancy in the 1995 and 1996 data (and hence the size of the correction required for losses due to multiple hodoscope hits), and the presence of the additional S3 layers provided additional background rejection.

\section{B. Comparisons with Other Experiments}

Experiment 878 has measured antiproton yields as a function of collision geometry in reactions of $\mathrm{Au}+\mathrm{Au}$ ions at $10.8 \mathrm{~A} \mathrm{GeV} / \mathrm{c}$ [12, 13]. There are two differences between the reaction system between E878 and E864: (1) the target in E864 is $\mathrm{Pb}$ and (2) the beam momentum in E864 is higher at $11.5 \mathrm{~A} \mathrm{GeV/c}$. The target difference is quite small and is neglected in this comparison. However, the production of antiprotons is near threshold for nucleon-nucleon collisions at these energies and so the beam momentum difference must be accounted for. We assume that the ratio of antiproton yields in $\mathrm{Au}+\mathrm{Pb}$ reactions at the two energies is proportional to the ratio of antiproton yields in $\mathrm{p}+\mathrm{p}$ reactions at the two energies. Unfortunately, there is no usable data on antiproton production in $\mathrm{p}+\mathrm{p}$ reactions covering this particular energy range. Therefore a parametrization of the production cross sections (derived from $\mathrm{p}+\mathrm{p}$ data at higher energies and $\mathrm{p}+\mathrm{Be}$ data from $\mathrm{E} 802$ at $14.6 \mathrm{GeV} / \mathrm{c}$ [3]) is employed. Using this parameterization, one expects the ratio of the antiproton production cross sections at the two energies to be 1.5. The E878 invariant multiplicities are scaled up by this value. By considering fits to higher energy $\mathrm{p}+\mathrm{p}$ data that do not include the E802 p+Be data at $14.6 \mathrm{GeV} / \mathrm{c}$ [14], we estimate that this energy scaling contributes an additional $15 \%$ systematic error on the overall normalization of the scaled E878 points.

Experiment 878 measures invariant multiplicities nominally at $p_{T}=0$ (which is really at transverse momenta less than $\sim 30-50 \mathrm{MeV} / \mathrm{c}$ ). Using the procedure previously outlined, we extrapolate the E864 measurements to $p_{T}=0$ and compare the E864 and E878 mea- 
surements in Figure 0. While the two experiments agree well for low multiplicity collisions a substantial disagreement develops for more central collisions. For 10\% central collisions, the E864 measurements at midrapidity are a factor of $\sim 3.2$ larger than the corresponding E878 data.

It should be noted that both experiments do not use precisely the same definition of centrality: E864 measures the multiplicity of charged particles produced in the collisions, while E878 measures the $\gamma$ multiplicity (mostly from $\pi^{0}$ decay). In order to properly compare the two experiments the multiplicity ranges for both experiments must be converted to a (somewhat model-dependent) parameter. To do this, we have chosen to show the integrated antiproton yield at $p_{T}=0$ versus the number of "first" nucleon-nucleon collions in each centrality range. In order to estimate the number of first collisions in each multiplicity range for the E864 data, a GEANT [9] simulation was used in conjunction with RQMD [18] $\mathrm{Au}+\mathrm{Pb}$ events to generate a trigger probability vs. impact parameter distribution for each multiplcicity region (see Figure 9). These distributions were then folded with distributions of the number of first collisions vs. impact parameter from a simple Glauber model calculation. A similar procedure was applied to the E878 data, using the results of a simulation of the E878 multiplicity array [15]. The results of this exercise (shown in Figure 10) demonstrates that the E864 and E878 centrality ranges are quite similar.

In Figure 8 we also compare measurements of the minimum-bias cross section for $\mathrm{Au}+\mathrm{Pb}$ collisons at 11.5 A GeV/c with E878 and E886 [16]. It should be noted that experiment E886 only measured antiprotons from minimum bias collisions and thus there is no comparison as a function of centrality. As expected by the comparison of the E864 data with E878 for the four different centrality regions, the minimum bias invariant multiplicities measured in E864 are substantially larger than those measured by E878 and E886. 


\section{Strange Antibaryon Feed-Down}

There is a scenario which can reconcile the E864 and E878 results. Some of the antiprotons measured by the various experiments may be the daughter product of weak decays of strange antibaryons $\left(\bar{\Lambda}, \overline{\Sigma^{+}}, \overline{\Sigma^{0}}, \overline{\Xi^{0}}, \overline{\Xi^{-}}, \bar{\Omega}\right)$. This process is referred to as "feeding down" from the strange antibaryons into the antiprotons. Due to the significantly different designs of the two experiments, they have different acceptances from these decay product antiprotons. There are a number of antihyperon $(\bar{Y})$ feed-down channels into the antiproton:

$$
\begin{gathered}
\bar{\Lambda} \rightarrow \bar{p}+\pi^{+} \quad(65 \% \text { B.F. }) \\
\overline{\Sigma^{0}} \rightarrow \bar{\Lambda}+\gamma \rightarrow \bar{p}+\pi^{+}+\gamma \quad(100 \% \times 65 \% \text { B.F. }) \\
\overline{\Sigma^{+}} \rightarrow \bar{p}+\pi^{0} \quad(52 \% \text { B.F. }) \\
\overline{\Xi^{0}} \rightarrow \bar{\Lambda}+\pi^{0} \rightarrow \bar{p}+\pi^{+}+\pi^{0} \quad(99 \% \times 65 \% \text { B.F. }) \\
\overline{\Xi^{-}} \rightarrow \bar{\Lambda}+\pi^{+} \rightarrow \bar{p}+\pi^{+}+\pi^{+} \quad(99 \% \times 65 \% \text { B.F. })
\end{gathered}
$$

and multiple decay modes for the $\bar{\Omega}$. The decay of the $\overline{\Sigma^{0}}$ will produce additional $\bar{\Lambda}$ 's which will be indistinguishable from those created in the primary collision. The decay of the $\bar{\Lambda}$ and the $\overline{\Sigma^{+}}$will produce $\bar{p}$ 's whose production vertices do not coincide with the location of the primary interaction between the two nuclei. Therefore, the degree to which $\bar{p}$ 's from these decays contribute to a measurement of $\bar{p}$ production will vary among experiments.

Due to its large acceptance, the E864 spectrometer will detect $\bar{p}$ 's from $\bar{Y}$ decay. E864 does not have sufficient vertical resolution to distinguish $\bar{p}$ 's from $\bar{Y}$ decay based on the vertical projection of a particle to the target, and the analysis cuts do not preferentially reject antiprotons from $\bar{Y}$ decay. Therefore, the $\bar{p}$ 's detected in E864 are a combination of primary $\bar{p}$ 's and $\bar{p}$ 's from $\bar{Y}$ decay, in a ratio that reflects their production ratio. 
The E878 collaboration have also evaluated the acceptance of their spectrometer for feeddown from $\bar{Y}$ decay. At midrapidity the acceptance for $\bar{p}$ 's from $\bar{\Lambda}$ and $\overline{\Sigma^{0}}$ decay is $14 \%$ of the spectrometer acceptance for primordial $\bar{p}$ 's, and $10 \%$ of the $\bar{p}$ acceptance for $\overline{\Sigma^{+}}$decays [13]; the acceptance grows at higher rapidity. In what follows, we assume a uncertainty of $\pm 1 \%$ in the $\mathrm{E} 878$ acceptances for feed-down.

Since both E878 and E864 measure a different combination of primordial $\bar{p}$ production and feed-down from $\bar{Y}$ decay, we can in principle separate the two components if we make two explicit assumptions: both E864 and E878 understand their systematic errors, and the entire difference between the two experiments can be attributed to antihyperon feed-down. It is important to note that in energy scaling the E878 results we have implicitly assumed that the $\bar{Y}$ 's scale with energy in the same way as the $\bar{p}$ 's.

Given an understanding of the errors involved, we can perform a statistical analysis of the $\bar{Y} / \bar{p}$ ratio as a function of the E864 and E878 measurements (see Figures 11 and 12). This analysis results in the following limits on the ratio of $\bar{Y} / \bar{p}$ :

$$
\left(\frac{\bar{Y}}{\bar{p}}\right)_{\substack{y=1.6 \\
p_{T}=0}} \approx\left(\frac{\bar{\Lambda}+\overline{\Sigma^{0}}+1.1 \overline{\Sigma^{+}}}{\bar{p}}\right)>(98 \% \text { C.L. })\left\{\begin{array}{l}
0.02(100-70 \%) \\
0.10(70-30 \%) \\
1.0(30-10 \%) \\
2.3(10 \%) \\
0.2 \text { (minimum bias) }
\end{array}\right.
$$

while the most probable value of this ratio is $\sim 3.5$ for $10 \%$ central collisions. The factor of 1.1 multiplying the $\overline{\Sigma^{+}}$arises due to the different branching ratio and acceptance for the $\overline{\Sigma^{+}}$compared to the $\bar{\Lambda}$. The probability distributions in Figure 11 were generated using the measured E864 and E878 invariant multiplicities for each centraility bin. The statistical errors on these measurements were treated as Gaussian, while systematic errors on the measurements, energy scaling, $p_{T}=0$ extrapolation, and the E878 acceptance for feed-down were treated as definining a limit around the measured values.

E878 has not explicitly calculated their experimental acceptance for the doubly strange 
$\bar{\Xi}$ and the $\bar{\Omega}$, and thus they are not explicitly included in the above formula. These heavier strange antibaryons are generally thought to be further suppressed and thus a small contribution. However, in light of the unexpectedly large "feed-down" contributions from strange antibaryons, one should be careful not to neglect their contribution to this ratio.

This comparison indicates a $\bar{Y} / \bar{p}$ ratio in $\mathrm{Au}+\mathrm{Pb}$ collisions that is significantly greater than one at midrapidity and $p_{T}=0$. It should be noted that if the $\bar{Y}$ 's and the $\bar{p}$ are produced with different distributions in $y$ and $p_{T}$, then the ratio of integrated yields of these particles will differ from the ratio at central rapidity and $p_{T}=0$. Preliminary results from $\mathrm{Si}+\mathrm{Au}$ collisions based on direct measurements of $\bar{p}$ and $\bar{\Lambda}$ production by the E859 collaboration also indicate a ratio of integrated yields greater than one [19]. For comparison, the $\bar{\Lambda} / \bar{p}$ ratio in pp collisions at similar energies is $\sim 0.2[20]$.

An enhancement of antihyperons arises naturally in models that include a QGP, and therefore enhanced antimatter and strangeness production [21, 囵. Thermal models that use a temperature and baryon chemical potential derived from measured particle spectra also indicate that the $\bar{Y} / \bar{p}$ ratio could be larger than one [22]. However, extremely large values of the $\bar{Y} / \bar{p}$ are difficult to achieve in a thermal model unless the freezout temperature and/or $K^{+} / K^{-}$are pushed beyond experimentally observed values. Transport models such as RQMD [18 predict the $\bar{Y} / \bar{p}$ ratio to be less than one. Including in a cascade model conversion reactions such as

$$
\bar{p}+K^{+} \rightarrow \pi+\bar{\Lambda}
$$

and a lower annihilation cross section for the $\bar{\Lambda}$ relative to the $\bar{p}$ enhances the $\bar{Y} / \bar{p}$ ratio substantially [23]. However, such a model does not reproduce the trend with centrality seen in the E864 data.

\section{ANTIMATTER CORRELATIONS}




\section{A. Double Antiproton Events}

In the large sample of events from the 1995 ( "-0.75T") run with a single antiproton within the experimental acceptance, there are some events with two identified antiprotons in the same event. These two antiproton events give insight into the possible correlated production of antimatter. Since the number of individual nucleon-nucleon collisions in each $\mathrm{Au}+\mathrm{Pb}$ collision is large, if the sample of central events are similar in nature, the production of one antiproton should have very little relation to the production of a second antiproton.

In the 1995 data set there are approximately 43,000 antiprotons with rapidity less than 2.2, which were considered for this study. After corrections for background contributions, we find there are 3.8 events with two antiprotons.

If we assume that the production of antiprotons is uncorrelated, we can calculate the number of two antiproton events expected. One can think of the nucleus-nucleus collision as many $(n)$ nucleon-nucleon collisions each with a probability $(p)$ of producing an antiproton. Since the probability $(p)$ is small and the number of collisions $(n)$ is large, we calculate the probability of producing two antiprotons in the same event using Poisson statistics. The probability of producing one antiproton is:

$$
\operatorname{Prob}(1)=\text { Rate }_{\text {Singles }}=\mathrm{n} \times \mathrm{p}
$$

The probability of producing two antiprotons is:

$$
\operatorname{Prob}(2)=\frac{\text { Rate }_{\text {singles }}^{2}}{2}
$$

Since we have measured the rate of single antiprotons into our detector Rate Singles, $_{\text {, we calcu- }}$ late the expected number of two antiproton events at 1.8. The $90 \%$ confidence level upper limit on this number is five, which includes the experimentally measured value.

Given the agreement with the assumption of uncorrelated production, there are limits we can set on the possible correlated production of antimatter. We postulate that there are two distinct classes of events within the $10 \%$ central $\mathrm{Au}+\mathrm{Pb}$ sample: One class of purely hadronic reactions and one class with the formation of the quark-gluon plasma (QGP). 
In Figure 13 the predicted number of two antiproton events as a function of the fraction

of QGP events $f_{Q G P}$ and the antimatter enhancement factor $\epsilon$ is shown. The area in the dark box is where the predicted number of two antiproton events is greater than five and thus ruled out by the data at the $90 \%$ confidence level. If the QGP enhancement factor is small, the two antiproton yield is not changed significantly. Also, if most of the events are QGP, then regardless of the enhancement, there is no predicted increase in the two antiproton yield. However, if there is a large enhancement $(\epsilon>10)$ and the fraction of QGP events is between $5 \%$ to $25 \%$, the yield of two antiprotons is significantly increased. These specific scenarios are ruled out by this measurement at the $90 \%$ confidence level.

\section{B. Antideuteron Search}

We have performed a search for antideuterons using the 1995 data set of central $\mathrm{Au}+$ $\mathrm{Pb}$ interactions taken at the "- $0.75 \mathrm{~T}$ " magnetic field setting optimized for the acceptance of antideuterons. After processing the data, any tracked particle of charge negative one, rapidity $y<2.4$, passing all track quality $\chi^{2}$ selections, and having a reconstructed mass in the range $1.3<m<5 \mathrm{GeV} / \mathrm{c}^{2}$ is considered a possible antinuclei candidate. The mass distribution of these candidates is shown in Figure 14. The distribution is well fit by an exponential and has no significant signal at the antideuteron mass $m=1.874 \mathrm{GeV} / \mathrm{c}^{2}$.

The experiment is able to reduce this background through an energy measurement using our full coverage hadronic calorimeter. The calorimeter measures the deposited kinetic energy of hadrons in addition to the annihilation energy for antibaryons. The background processes expected to create high mass candidates in the tracking reconstruction are the result of neutrons which charge exchange in the vacuum exit window or air and produce a forward going proton traversing the downstream spectrometer. The protons have reasonable rapidity values, but reconstruct to erroneously large rigidities resulting from the incorrect assumption that the particle originated at the target. These candidates should leave significantly less energy in the calorimeter than expected if they are actually protons compared 
with real antideuterons or heavier antinuclei.

Since these candidates are all assumed to be antimatter, the reconstructed calorimeter mass must account for the annihilation contribution. In studies of antiproton showers from test beam data and from the 1995 data, it was observed that only $\approx 84 \%$ of the annihilation energy was recorded in the calorimeter. Thus, the calorimeter mass formula is modified to reflect this loss:

$$
\operatorname{mass}=\frac{E}{\gamma+0.68}
$$

The tracking mass resolution is $\frac{\Delta m}{m} \simeq 5 \%$, which yields a $\sigma_{m}=0.094 \mathrm{GeV} / \mathrm{c}^{2}$ for antideuterons. The distribution of calorimeter masses for candidates whose tracking mass is within $\pm 2 \sigma_{m}$ of the antideuteron mass $\left(1.687<m<2.061 \mathrm{GeV} / \mathrm{c}^{2}\right)$ is shown in Figure 15 . One can see the peak mean value is less than $0.938 \mathrm{GeV} / \mathrm{c}^{2}$. Protons have a lower calorimeter mass $\left(<0.938 \mathrm{GeV} / \mathrm{c}^{2}\right)$ when calculated using Equation 12 since they do not deposit any energy beyond their kinetic energy (there is no annihilation energy contribution). The background candidates appear to be protons as expected from charge exchange background. Most protons should not reconstruct such a large antimatter mass and fire the late-energy trigger. However, the calorimeter energy response has a non-Gaussian high side tail. These candidates are protons which occupy the high side tail part of the energy response distribution. As can be seen in the plot, the calorimeter is a powerful tool for rejecting this proton background. A cut is then placed on calorimeter mass being greater than $1.600 \mathrm{GeV} / \mathrm{c}^{2}$.

If one assumes that all of the observed candidates are from charge exchange background (really protons striking the calorimeter), then the background shape can be fit. The tracking mass distribution with no cut on the calorimeter mass is fit to a simple exponential function as shown in Figure 14. If the candidates are all protons striking the calorimeter, the calorimeter mass distribution should be the same regardless of the tracking mass. Thus, one can use the exponential function fit parameters from the tracking mass distribution with no calorimeter cuts to describe the tracking mass distribution with a calorimeter mass cut.

The tracking mass distribution is plotted in Figure 14 with a cut on the calorimeter 
mass greater than $1.600 \mathrm{GeV} / \mathrm{c}^{2}$. There are ten candidates within the $\pm 2 \sigma_{m}$ range of the antideuteron. The background fit distribution shown in Figure 14 is renormalized to the total number of counts and plotted. The exponential fit seems a reasonable description of the distribution. The total number of counts from the fit in the region of the antideuteron (within $\left.\pm 2 \sigma_{m}\right)$ is 9.0. Thus, there is no significant signal above background for the antideuteron.

One can then ask, how many real antideuterons would there have to be to make a statistically significant peak above the background distribution. There are nine predicted background events, and thus the Poisson statistics 90\% confidence level upper limit is 14.2. If more than 14.2 candidates were observed within the antideuteron mass range, there is less than a $10 \%$ chance that it is due to a statistical fluctuation in the background events. Thus, we set the $90 \%$ confidence level upper limit on antideuteron production at $N_{\text {Poisson }}-$ $N_{\text {Background }}=5.2$.

In order to translate this Poisson statistics limit into a total upper limit on the production of antideuterons, the various acceptances and efficiencies must be known. It is also possible using a specific production model to set the $90 \%$ confidence level upper limit on the invariant multiplicity in a given region of momentum space. In the discussion that follows, we will asuume a model in which the production in $p_{T}$ and rapidity $(y)$ can be factored as:

$$
\frac{1}{2 \pi p_{T}} \frac{d N}{d y d p_{T}}=A_{0} e^{-2 p_{T} /<p_{T}>} e^{-\left(y-y_{c m}\right)^{2} / 2 \sigma_{y}^{2}}
$$

We have assumed a rapidity width $\sigma_{y}=0.5$ and a mean transverse momentum $<p_{T}>=1.00$ $\mathrm{GeV} /$ c. Using this production model, we set a $90 \%$ confidence level upper limit on the production of antideuterons at $2.78 \times 10^{-7}$ per $10 \%$ central $\mathrm{Au}+\mathrm{Pb}$ interaction.

One can relate the limit over all phase space to the limit on the invariant multiplicity $\left(A_{0}\right)$ at midrapidity and $p_{T}=0$.

$$
A_{0}=\frac{N_{\text {TotalLimit }}}{(2 \pi)^{3 / 2} \sigma_{y} \frac{\leq p_{T}>^{2}}{4}}
$$

The upper limit on the invariant multiplicity at midrapidity $y=1.6$ and $p_{t}=0$ is $1.4 \times$ $10^{-7} \mathrm{GeV}^{-2} \mathrm{c}^{2}$. We have tested the model dependency of these upper limits and find that 
with extreme ranges of production models, one can vary the upper limits by approximately $\pm 50 \%$.

Using our antiproton measurements and these upper limits, we calculate the $90 \%$ confidence level upper limits on the coalescence scale factor $\overline{B_{2}}$ for antideuterons. This scale factor may be a function of where in momentum space the measurement is made, thus we give the limit at midrapidity $(y=1.6)$ and $p_{t}=0$. Our measured invariant multiplicity for antiprotons is $1.16 \times 10^{-2} \mathrm{GeV}^{-2} \mathrm{c}^{2}$ (from the combined $10 \%$ central 1995 and 1996 data). The upper limit on the invariant multiplicity for antideuterons is $1.41 \times 10^{-7} \mathrm{GeV}^{-2} \mathrm{c}^{2}$. The upper limit on the scale factor is:

$$
\overline{B_{2}}=\frac{\left[\frac{1}{2 \pi p_{t}} \frac{d^{2} N}{d y d p_{t}}(\bar{d})\right]}{\left[\frac{1}{2 \pi p_{t}} \frac{d^{2} N}{d y d p_{t}}(\bar{p})\right]^{2}} \leq 1.0 \times 10^{-3} \mathrm{GeV}^{2} \mathrm{c}^{-2}
$$

This upper limit is shown as an arrow in Figure 16, along with a comparison to coalescence scale factors measured at Bevelac and SPS energies [24] [25] [26].

This scale factor is significantly below the global value of $1.2 \times 10^{-2} \mathrm{GeV}^{2} \mathrm{c}^{-2}$ predicted by the "simple" coalescence model. However, since this prescription has failed to describe systems where the collision volume is expected to be large compared with the deuteron/antideuteron size [27], it is not surprising that it is in disagreement with the value obtained here.

If the source distribution of antinucleons has a similar spatial extent as the nucleon source, then the scale factor for deuterons $B_{2}$ is expected to be the same as for antideuterons $\overline{B_{2}}$. Recently, E864 has presented measurements of protons and deuterons around midrapidity and low transverse momentum. The scale factor from the analysis of E864 light ion data [28] is also shown in Figure 16.

$$
B_{2}=1.1 \pm .4 \times 10^{-3} \mathrm{GeV}^{2} \mathrm{c}^{-2}
$$

The uncertainties are dominated by systematic errors in the deuteron and proton invariant multiplicities. This measured scale factor is at the same level as the upper limit for the antideuteron scale factor. We cannot determine whether $\overline{B_{2}}$ is significantly lower than $B_{2}$. 
Thus, it is impossible to comment on whether the rate of antideuteron production is smaller due to preferential surface emission of antimatter.

If we consider the most probable value of the ratio $(\bar{Y}) / \bar{p}=3.5$ for $10 \%$ central collisions, the primordial antiproton multiplicity at midrapidity and $p_{t}=0$ should be a factor of $\sim 3.3$ lower than measured in E864. In this picture, the 90\% confidence level upper limit on the coalescence scale factor would be

$$
\overline{B_{2}} \leq 1.1 \times 10^{-2} \mathrm{GeV}^{2} \mathrm{c}^{-2}
$$

This value is approximately at the level measured in $\mathrm{p}+\mathrm{A}$ collisions, in accord with the "simple" coalescence model, where the collision volume is expected to be quite small. Thus,

if the $\bar{Y}$ production is correctly calculated, the limit set on antideuteron production is not very significant in the context of coalescence models.

There have been two previous measurements of antideuteron production in heavy ion collisions. The first was from the E858 experiment which observed two antideuterons in minimum bias $\mathrm{Si}+\mathrm{Au}$ collisions at $14.6 \mathrm{~A} \mathrm{GeV} / \mathrm{c}$ 29. They calculated a coalescence factor of approximately $\overline{B_{2}} \leq 1.0 \times 10^{-3} \mathrm{GeV}^{2} \mathrm{c}^{-2}$. While this value is consistent with our observation, it is difficult to make any direct comparison since the E858 value is for minimum bias collisions involving a much smaller projectile. The second measurement is from experiment $\mathrm{NA} 52$ in $\mathrm{Pb}+\mathrm{Pb}$ central collisions at $160 \mathrm{~A} \mathrm{GeV} / \mathrm{c}$ [30]. They observe a coalescence factor of approximately $\overline{B_{2}} \approx 5.0 \pm 3.0 \times 10^{-4} \mathrm{GeV}^{2} \mathrm{c}^{-2}$. They also find the factor for deuterons $B_{2}$ is the same within statistical and systematic errors. While our upper limit on antideuterons is consistent with their value, our deuteron coalescence factor is somewhat higher. This observation is not surprising due to larger source dimensions in the higher energy collisions studied by NA52.

\section{CONCLUSIONS}

We have presented results from Experiment 864 for antiproton production and antideuteron limits in $\mathrm{Au}+\mathrm{Pb}$ collisions at $11.5 \mathrm{GeV} / \mathrm{c}$ per nucleon. We have measured in- 
variant multiplicities for antiprotons above midrapidity and at low transverse momentum as a function of collision geometry. These measurements are within systematic errors of our previously reported results [11], and, when compared with the results from Experiment 878, may indicate a significant contribution to the measured antiproton yield from the decay of strange antibaryons.

We have also studied correlated production of antimatter using events with more than one antiproton and a search for antideuterons. For antideuterons we see no statistically significant signal. We set upper limits on the production at approximately $3 \times 10^{-7}$ per $10 \%$ highest multiplicity $\mathrm{Au}+\mathrm{Pb}$ interaction. 


\section{REFERENCES}

* $\quad$ Present address: Vanderbilt University, Nashville, Tennessee 37235

$\dagger \quad$ Present Address: Anderson Consulting, Hartford, CT

$\ddagger \quad$ Present address: Univ. of Denver, Denver CO 80208

$\S \quad$ Deceased.

"| Present address: McKinsey \& Co., New York, NY 10022

ฯ Present address: Department of Radiation Oncology, Medical College of Virginia, Richmond VA 23298

** Present address: Columbia University, Nevis Laboratory, Irvington, NY 10533

${ }^{\dagger} \quad$ Present address: University of Tennessee, Knoxville TN 37996

执 Present address: Institut de Physique Nucléaire, 91406 Orsay Cedex, France

$\S \S \quad$ Present Address: Institute for Defense Analysis, Alexandria VA 22311

"I\| Present Address: MIT Lincoln Laboratory, Lexington MA 02420-9185

[1] P. Koch, B. Müller, H. Stöcker and W. Greiner, Modern Physics Letters A 8737 (1988); J. Ellis, U. Heinz and Henry Kowalski, Phys. Lett. B 233223 (1989); U. Heinz, P. R. Subramanian, H. Stöcker and W. Greiner, Journal of Physics G: Nuclear Physics 12, 1237 (1986).

[2] V. Koch, G. E. Brown and C. M. Ko, Phys. Lett. B 26529 (1991); J. Schaffner, I. N. Mishustin, L. M. Satarov, H. Stöcker and W. Greiner, Z. Phys. A 34147 (1991); P. Koch and C. B. Dover, Phys. Rev. C 40 (1989) 145; S. Gavin, M. Gyulassy, M. Plümer and R. Venugopalan, Phys. Lett. B 234175 (1990).

[3] S. H. Kahana, Y. Pang, T. Schlagel and C. B. Dover, Phys. Rev. C 471356 (1993). 
[4] J.L. Nagle, B.S. Kumar, M.J. Bennett, S.D. Coe, G.E. Diebold, and J.K. Pope, Phys. Rev. Lett. 732417 (1994).

[5] T. Armstrong et al., to be submitted to NIM.

[6] T. Armstrong et al., Nucl. Instr. Meth. A 406 (1998) 227.

[7] J. Hill et al., accepted for publication in NIM.

[8] P. Haridas, G. Van Buren, J. Tomasi, M.S.Z. Rabin, K. Barish, and R.D. Majka, Nucl. Instr. Meth. A 385 (1997) 412.

[9] GEANT Detector Description and Simulation Tool, CERN Program Library Long Writeup W5013.

[10] J. Nagle, Ph.D. Thesis, Yale University (1997).

[11] T. Armstrong et al., Phys. Rev. Lett. 793351 (1997); J. Lajoie, Ph.D. Thesis, Yale University (1996).

[12] D. Beavis et al., Phys. Rev. Lett. 753633 (1995).

[13] M. Bennett et al., Phys. Rev. C 561521 (1997).

[14] J. Costales, Ph.D. Thesis, Massachusetts Institute of Technology (1990)

[15] D. Beavis et al., Nucl. Instrum. Meth. A 357, 283 (1995).

[16] G.E. Diebold et al., Phys. Rev. C 482984 (1993).

[17] The E866 Collaboration, H. Sako et al., in Heavy Ion Physics at the AGS (HIPAGS 96), C. A. Pruneau, G. Welke, R. Bellweid, S. J. Bennett, J. R. Hall and W. K. Wilson Eds., WSU-NP-96-16, Wayne State University, Dec. 1996.

[18] H. Sorge, Phys. Rev. C 523291 (1995); M. Gonin et al., Phys. Rev. C 51310 (1995).

[19] The E859 Collaboration, Y. Wu et al., in HIPAGS 96, see ref [17]. 
[20] V. Blobel et al., Nuclear Physics B 69454 (1974); A. M. Rossi et al., Nuclear Physics B 84269 (1975).

[21] P. Koch, B. Muller, J. Rafelski, Phys. Rep. 142, 167 (1986).

[22] P. Braun-Munzinger, J. Stachel, J. P. Wessels and N. Xu, Phys. Lett. B 34443 (1995).

[23] G. J. Wang, G. Welke, R. Bellwied, and C. Pruneau, nucl-th/9807036

[24] S. Nagamiya et al., Phys. Rev. C 24, 971 (1981); P. Lemaire, Phys. Rev. Lett. 37, 667 (1976); M Anikina, JINR Report No. 1-84-216, Dubna (1984).

[25] W. Bozzoli, Nucl. Phys. B 144, 317 (1978).

[26] J.W. Cronin et al., Phys. Rev. D 11, 3105 (1975).

[27] J. Barrette et al., Phys. Rev. C 50, 1077 (1994); J.L. Nagle, B.S. Kumar, M.J. Bennett, S.D. Coe, G.E. Diebold, and J.K. Pope, Phys. Rev. Lett. 73, 1219 (1994)

[28] N. K. George, Ph. D. Thesis, Yale University (1998)

[29] A. Aoki et al., Phys. Rev. Lett. 69, 2345 (1992).

[30] G. Ambrosini et al., Nucl. Phys. A 638, 411c (1998). 


\section{TABLES}

\begin{tabular}{|c|c|}
\hline \hline rapidity & $\frac{1}{2 \pi p_{T}} \frac{d N}{d y d p_{T}}$ at $p_{T}=0\left(\times 10^{-2},{\left.\mathrm{in} \mathrm{GeV}^{-2} \mathrm{c}^{2}\right)}\right.$ \\
\hline \hline $1.8<y<2.0$ & $1.00 \pm 0.05$ \\
$2.0<y<2.2$ & $0.67 \pm 0.03$ \\
$2.2<y<2.4$ & $0.36 \pm 0.02$ \\
\hline \hline
\end{tabular}

TABLE I. Antiproton invariant multiplicities at $p_{T}=0$ from the $1995(-0.75 \mathrm{~T}) 10 \%$ central data. The errors listed are statistical only.

\begin{tabular}{|c|c|c|c|c|c|}
\hline \hline & \multicolumn{5}{|c|}{$\frac{1}{2 \pi p_{T}} \frac{d N}{d y d p_{T}}$ at $p_{T}=0\left(\times 10^{-2}, \mathrm{in}_{\mathrm{GeV}}^{-2} \mathrm{c}^{2}\right)$} \\
\hline rapidity & $100-70 \%$ & $70-30 \%$ & $30-10 \%$ & $10 \%$ & min. bias \\
\hline \hline $1.4<y<1.5$ & $0.12 \pm 0.02$ & $0.56 \pm 0.06$ & $0.87 \pm 0.08$ & $0.94 \pm 0.11$ & $0.48 \pm 0.02$ \\
$1.5<y<1.6$ & $0.14 \pm 0.01$ & $0.55 \pm 0.02$ & $0.98 \pm 0.04$ & $1.17 \pm 0.06$ & $0.51 \pm 0.01$ \\
$1.6<y<1.7$ & $0.13 \pm 0.01$ & $0.53 \pm 0.02$ & $0.90 \pm 0.03$ & $1.15 \pm 0.05$ & $0.48 \pm 0.01$ \\
$1.7<y<1.8$ & $0.12 \pm 0.01$ & $0.52 \pm 0.02$ & $0.80 \pm 0.02$ & $1.11 \pm 0.03$ & $0.46 \pm 0.01$ \\
$1.8<y<1.9$ & $0.11 \pm 0.01$ & $0.44 \pm 0.02$ & $0.75 \pm 0.02$ & $1.00 \pm 0.04$ & $0.39 \pm 0.01$ \\
$1.9<y<2.0$ & $0.093 \pm 0.005$ & $0.38 \pm 0.02$ & $0.68 \pm 0.02$ & $0.88 \pm 0.03$ & $0.34 \pm 0.01$ \\
$2.0<y<2.1$ & $0.060 \pm 0.004$ & $0.30 \pm 0.01$ & $0.51 \pm 0.02$ & $0.71 \pm 0.03$ & $0.27 \pm 0.01$ \\
\hline \hline
\end{tabular}

TABLE II. Antiproton invariant multiplicities at $p_{T}=0$ vs. centrality from the $1996(-0.45 \mathrm{~T})$ minimum-bias data. The data are listed by multiplicity regions used in the analysis. The invariant multiplicities for the full minimum-bias sample are also listed. The errors listed are statistical only. 


\section{FIGURES}

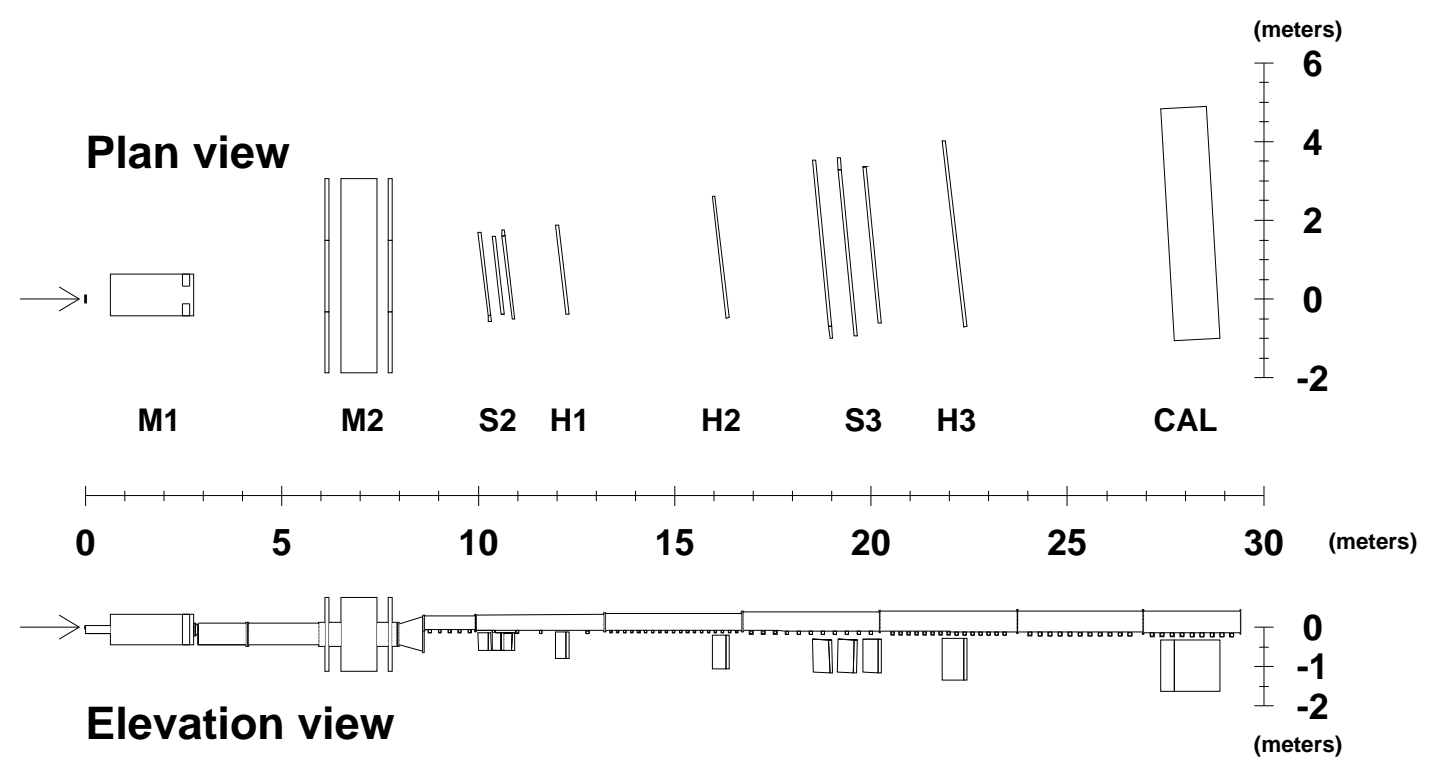

FIG. 1. The E864 detector in plan and elevation views, showing the dipole magnets M1 and M2, hodoscopes (H1, H2 and H3), straw tube arrays (S2 and S3) and hadronic calorimeter (CAL). The vacuum chamber is not shown in the plan view. 

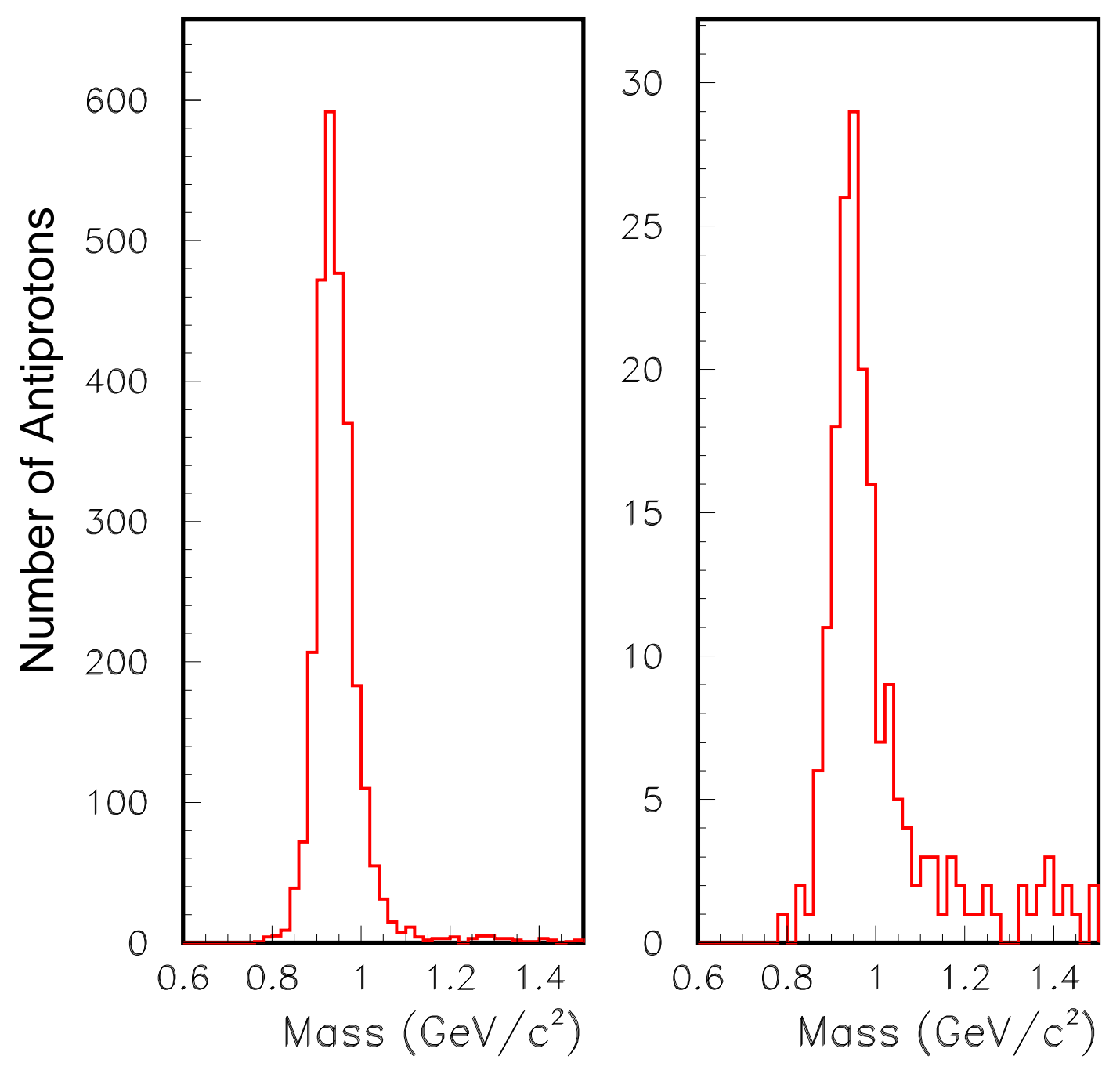

FIG. 2. Antiproton mass distributions for $Z=-1$ particles from the 1995 ("-0.75T") data set. The left panel show the mass distribution for particles that triggered the LET, while the right panel shows the mass distribution for particles that did not. These mass distributions are for particles with rapidity $1.8<y<2.0$ and transverse momentum $125<p_{T}<150 \mathrm{MeV} / \mathrm{c}$. 


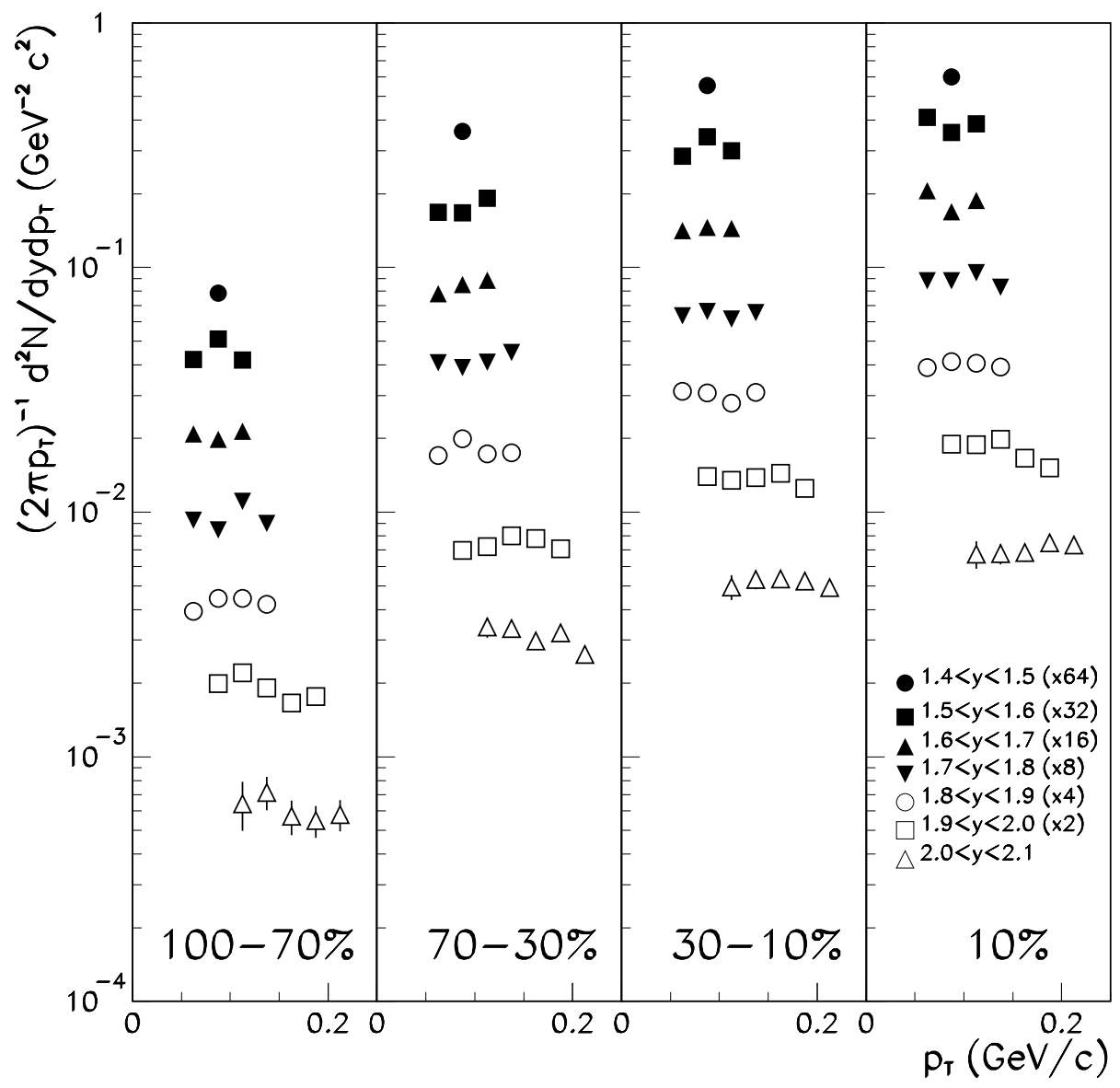

FIG. 3. Invariant multiplicities for antiprotons as measured in the four different centrality bins from the 1996 data. Note that the data are approximately flat in each rapidity interval over the measured range in transverse momentum. 


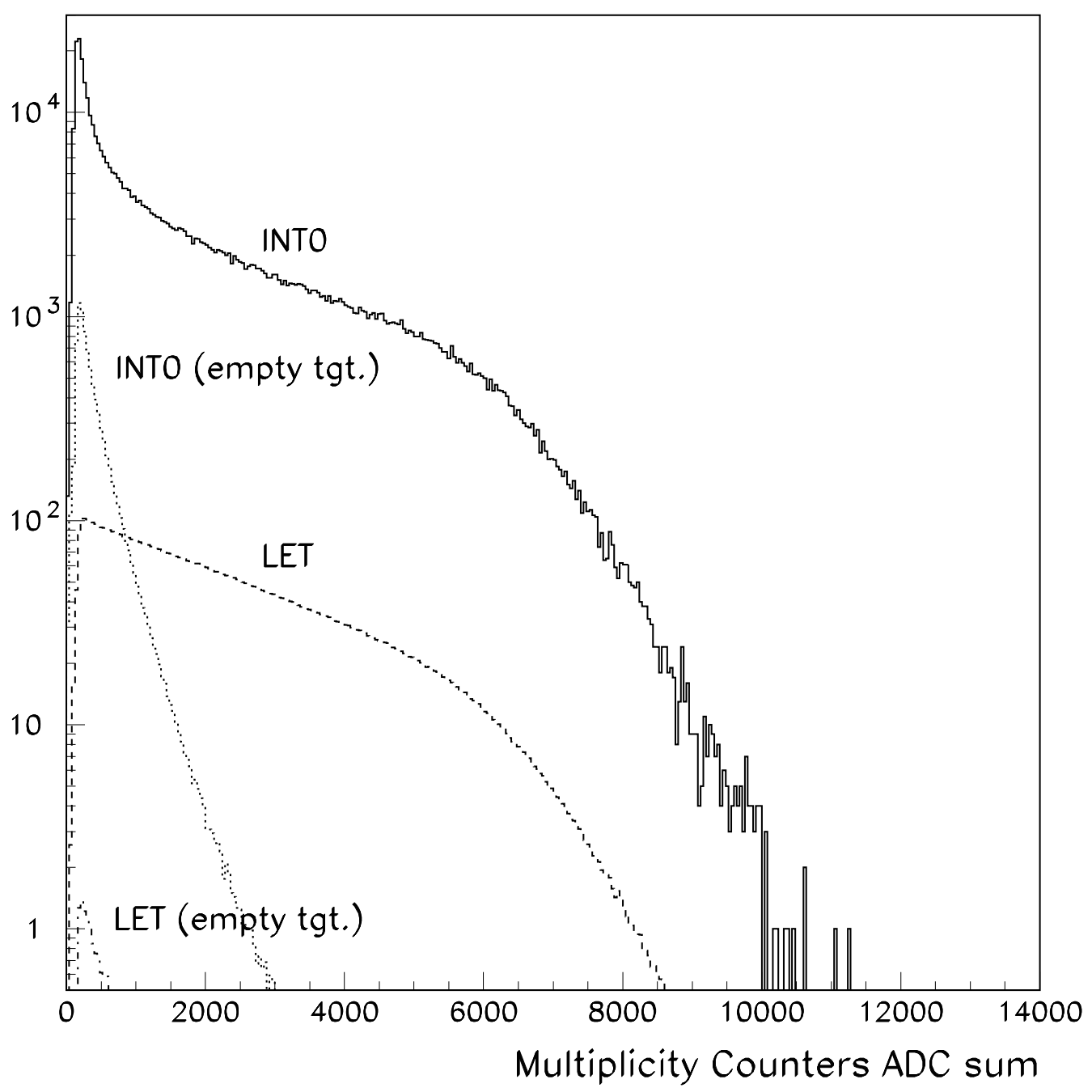

FIG. 4. Multiplicity counter ADC distributions for INT0 (minimum bias) and LET triggers for $10 \% \mathrm{~Pb}$ and empty targets; the distributions are scaled to the same number of incident $\mathrm{Au}$ ions. For LET triggers the empty target contribution is less that $1 \%$ of the distribution for the lowest multiplicities. 


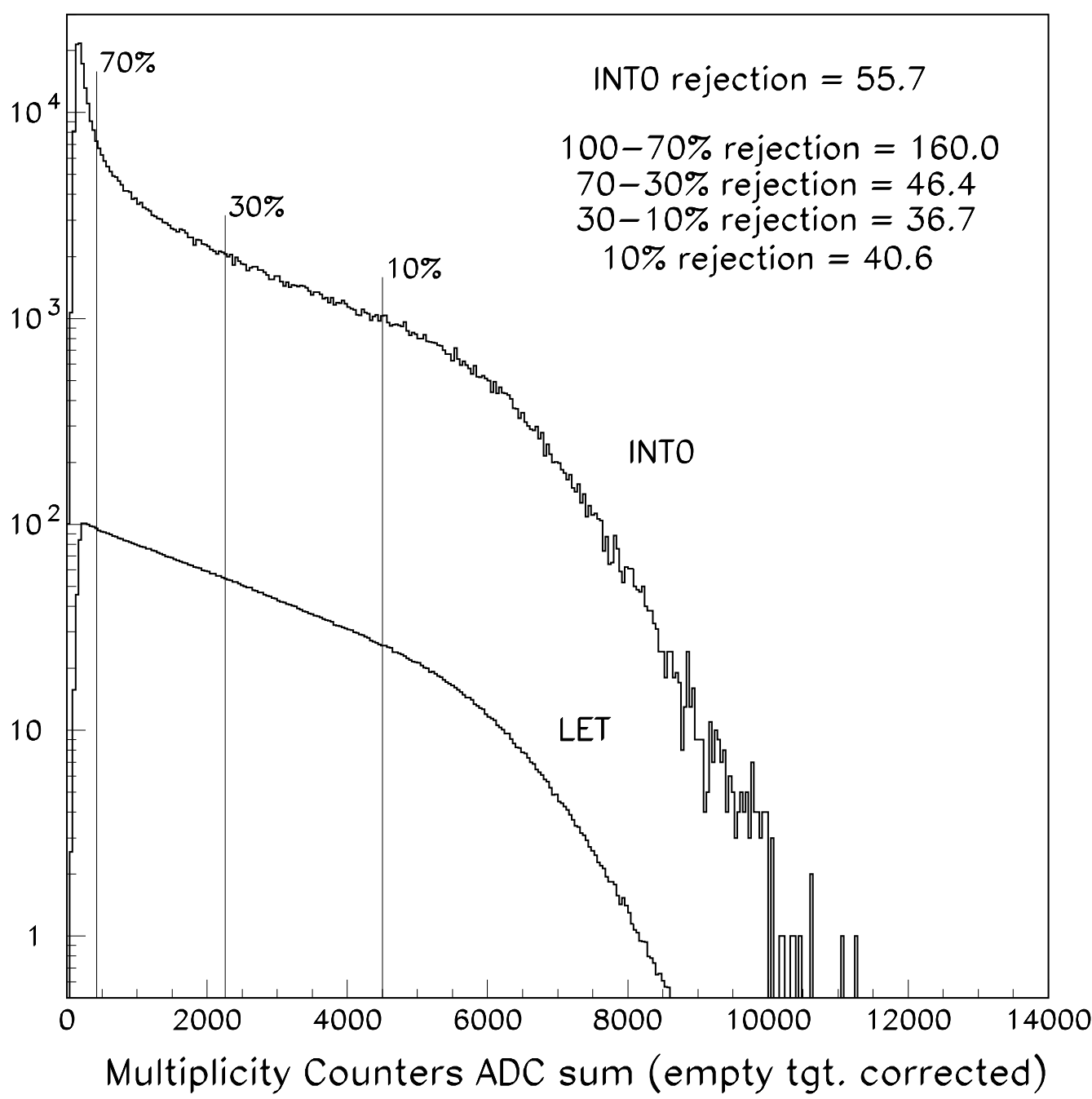

FIG. 5. Multiplicity counter ADC distributions (corrected for empty target contributions) showing the centrality cuts used in the analysis. The effective LET rejection factor for each multiplicity region is also shown. 


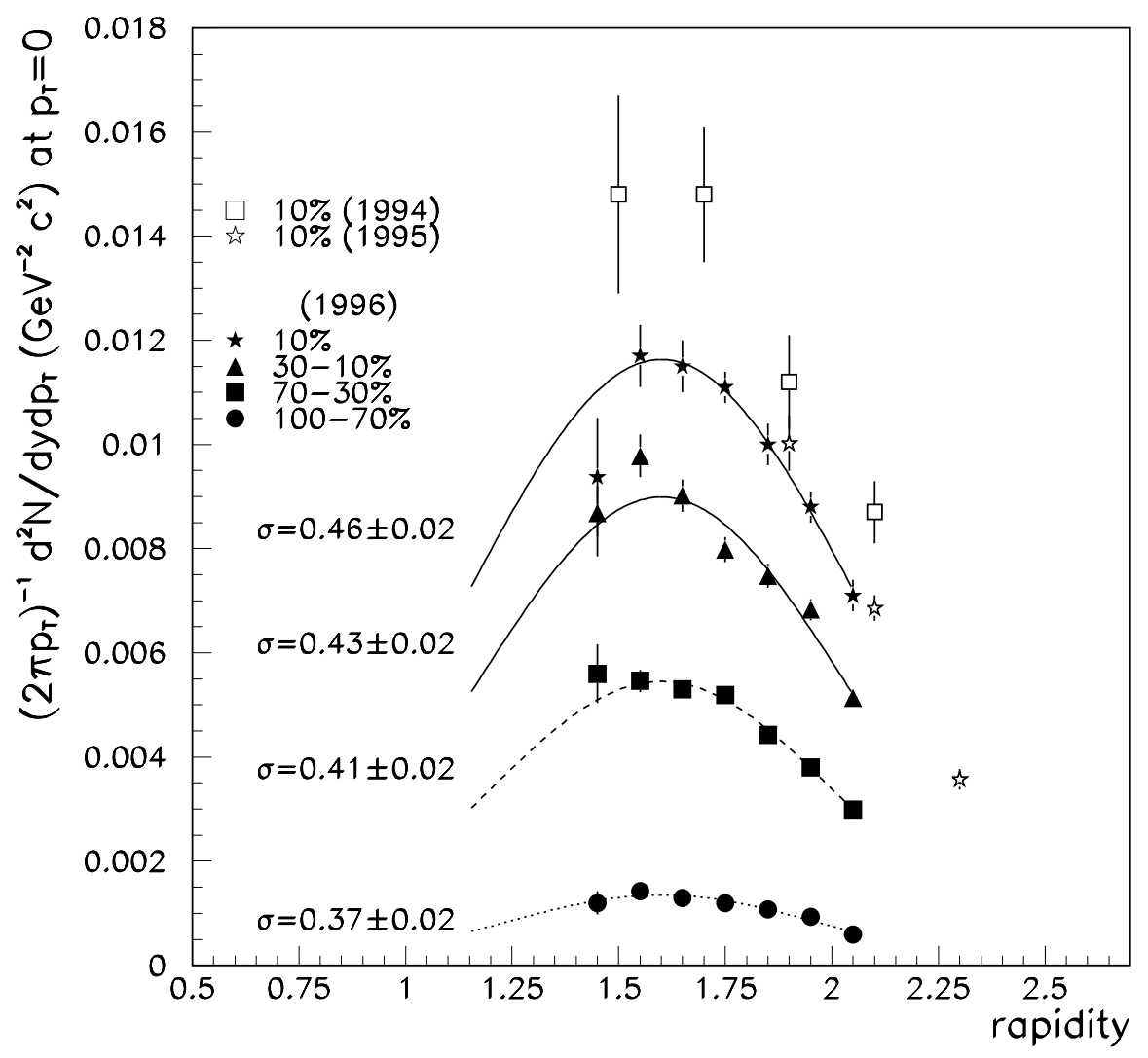

FIG. 6. Antiproton invariant multiplicities as a function of centrality showning the 1994, 1995 and 1996 data sets from E864. The error bars shown are statistical only. Systematic errors are estimated to be $20 \%$ on the 1994 data, $15 \%$ for the 1995 data, and $10 \%$ for the 1996 data, not including a 6 to $p_{T}=0$. The fits are constrained to have a mean value at midrapidity $(y=1.6)$. 


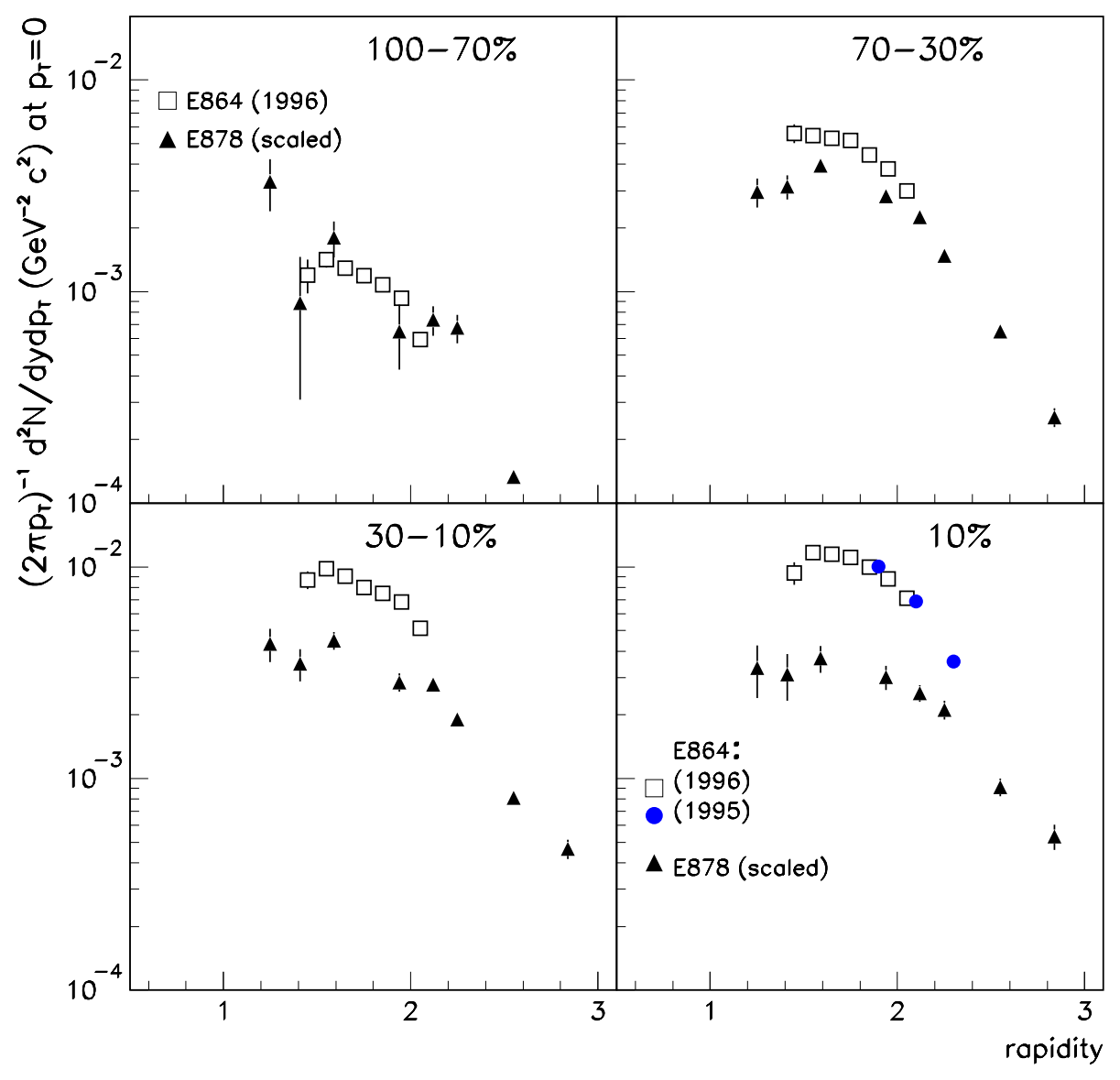

FIG. 7. Antiproton invariant multiplicities at $p_{T}=0$ compared to E878 as a function of centrality. Note that while the two experiments agree for peripheral collisions, they disagree markedly for more central collisions. The E878 data have been scaled up as described in the text to account for the lower beam momentum. 


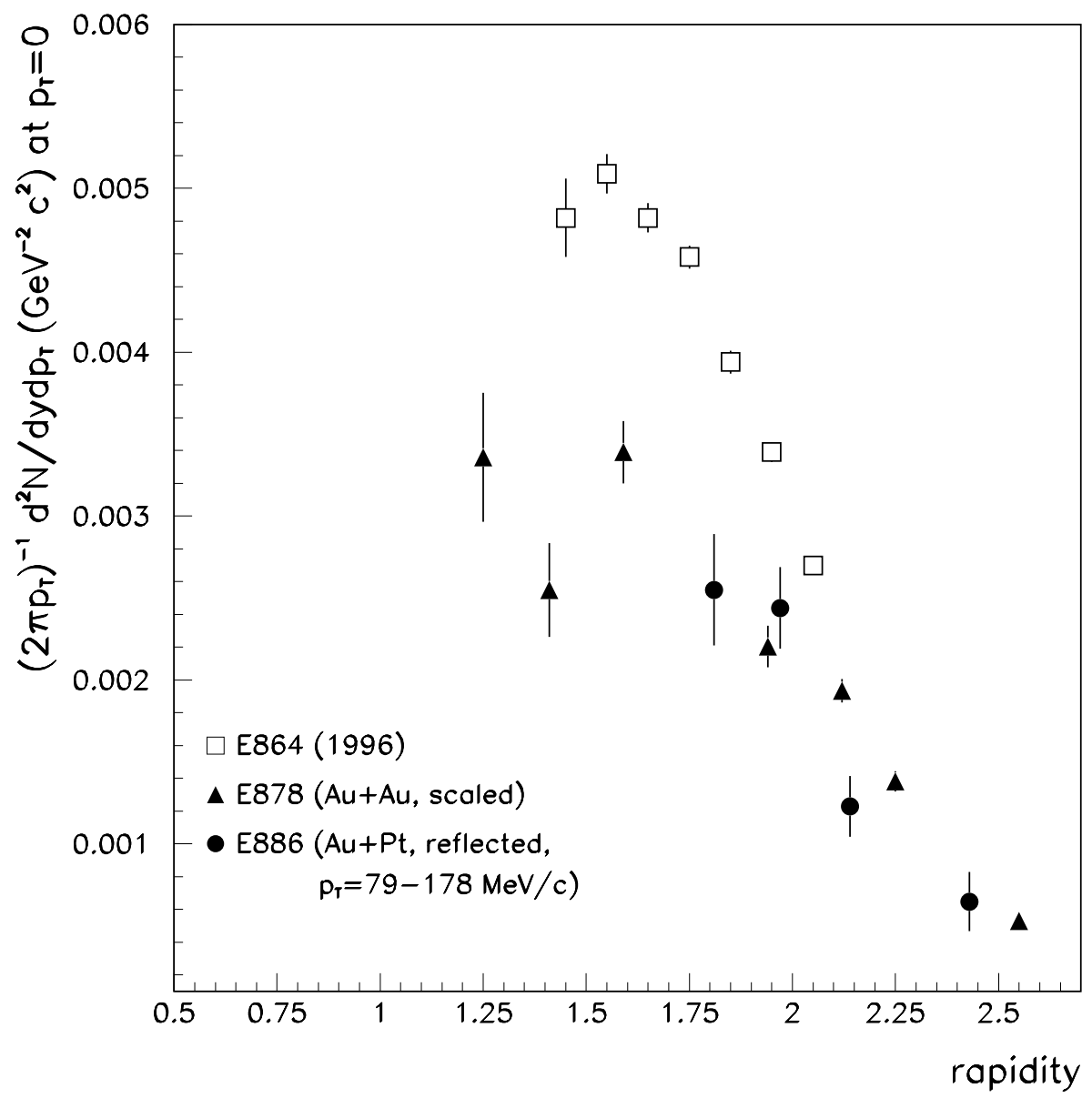

FIG. 8. Antiproton invariant multiplicities from the 1996 (-0.45T) minimum bias data. Also shown is minimum bias data from E878 (scaled up to $11.5 \mathrm{GeV} / \mathrm{c}$ ) and data from E886 (shown reflected about midrapidity, $y=1.6)$. 


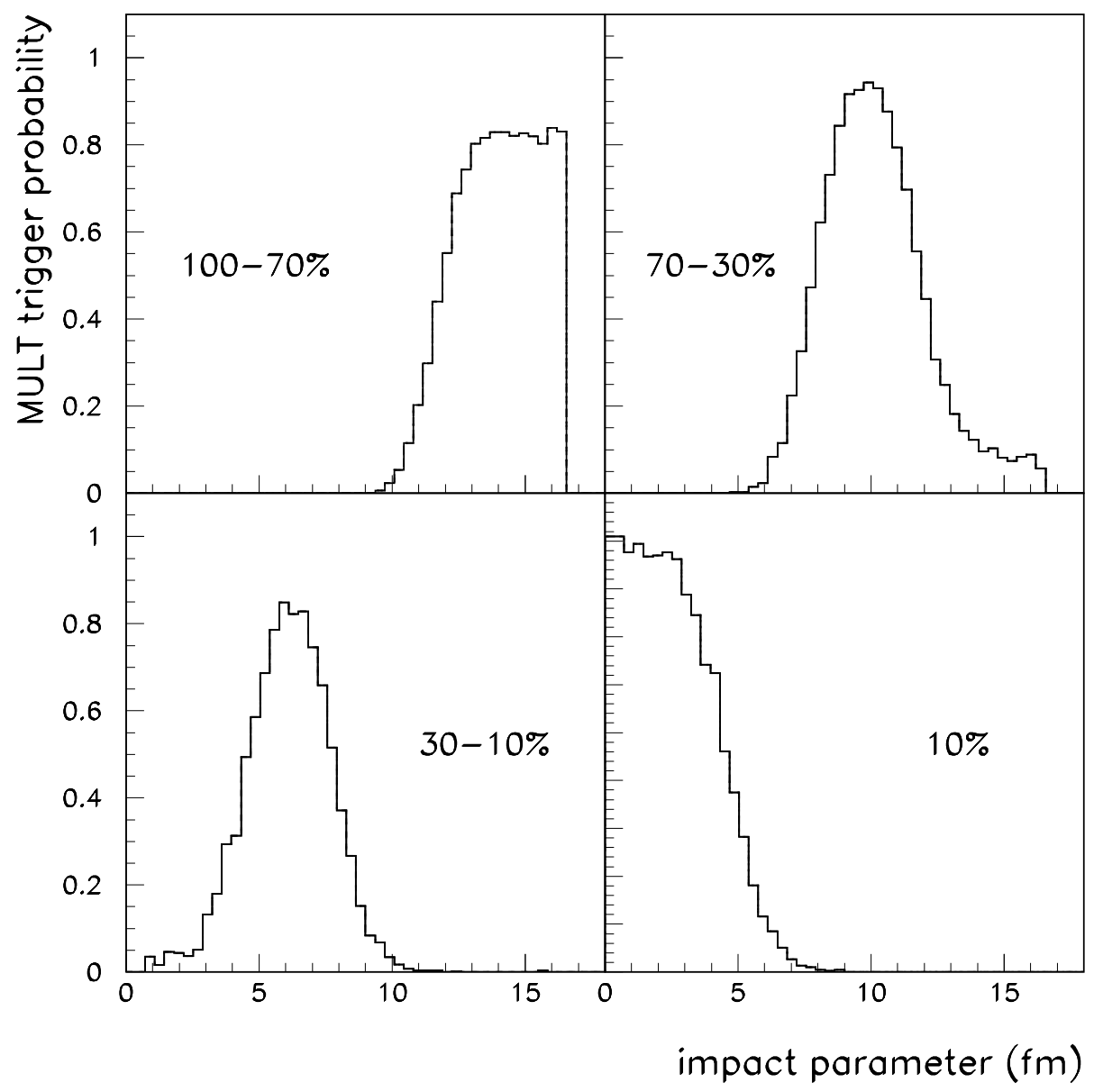

FIG. 9. Trigger probability vs. impact parameter for the E864 multiplicity counter in the four centrality regions used in the analysis of the 1996 data. These distributions were generated using RQMD events in a GEANT simulation of the E864 multiplicity counter. 


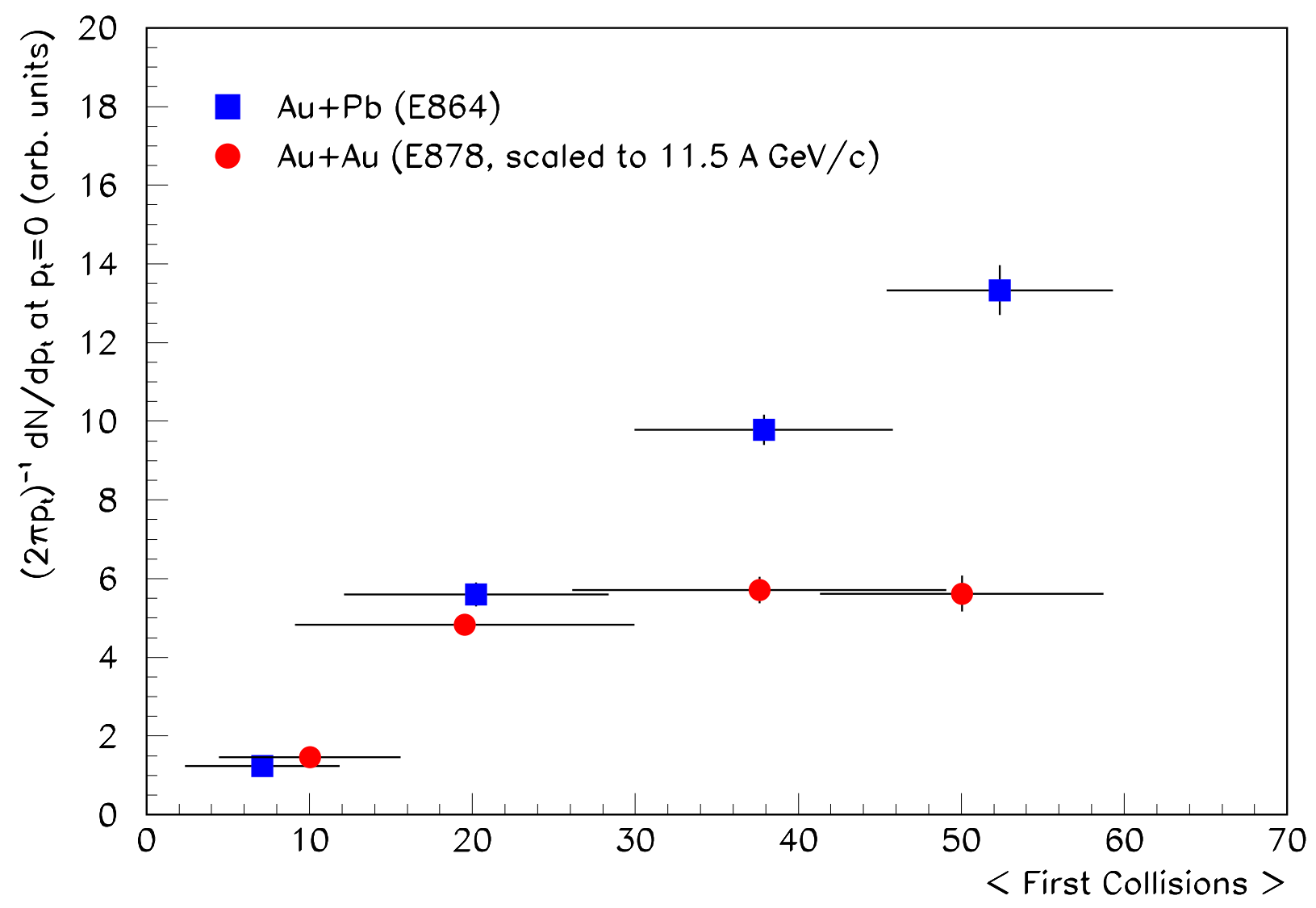

FIG. 10. Integrated antiproton yield at $p_{T}=0$ vs. the mean number of first collisions. The number of first collisions are generated using simulations of the detector response and a Glauber model; the horizontal bar for each multiplicity range indicates the RMS of the first collisions distribution. 

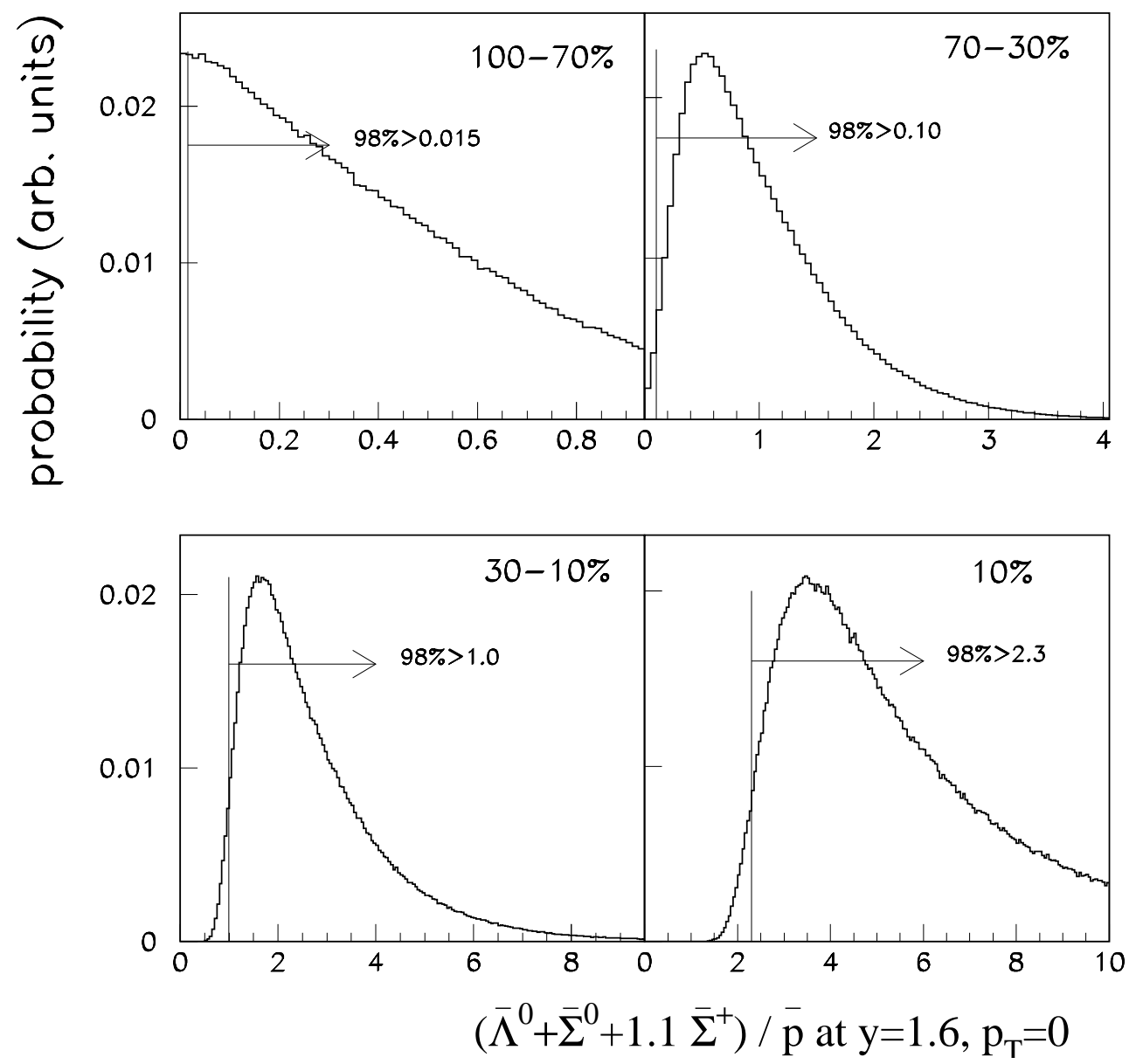

FIG. 11. Probability distributions for the antihyperon/antiproton ratio as a function of centrality. The distributions are generated from the ratio of the E864 antiproton measurements at midrapidity vs. the E878 measurements using the statistical and systematic errors. See the text for details. The $98 \%$ confidence levels are marked in for each distribution. 


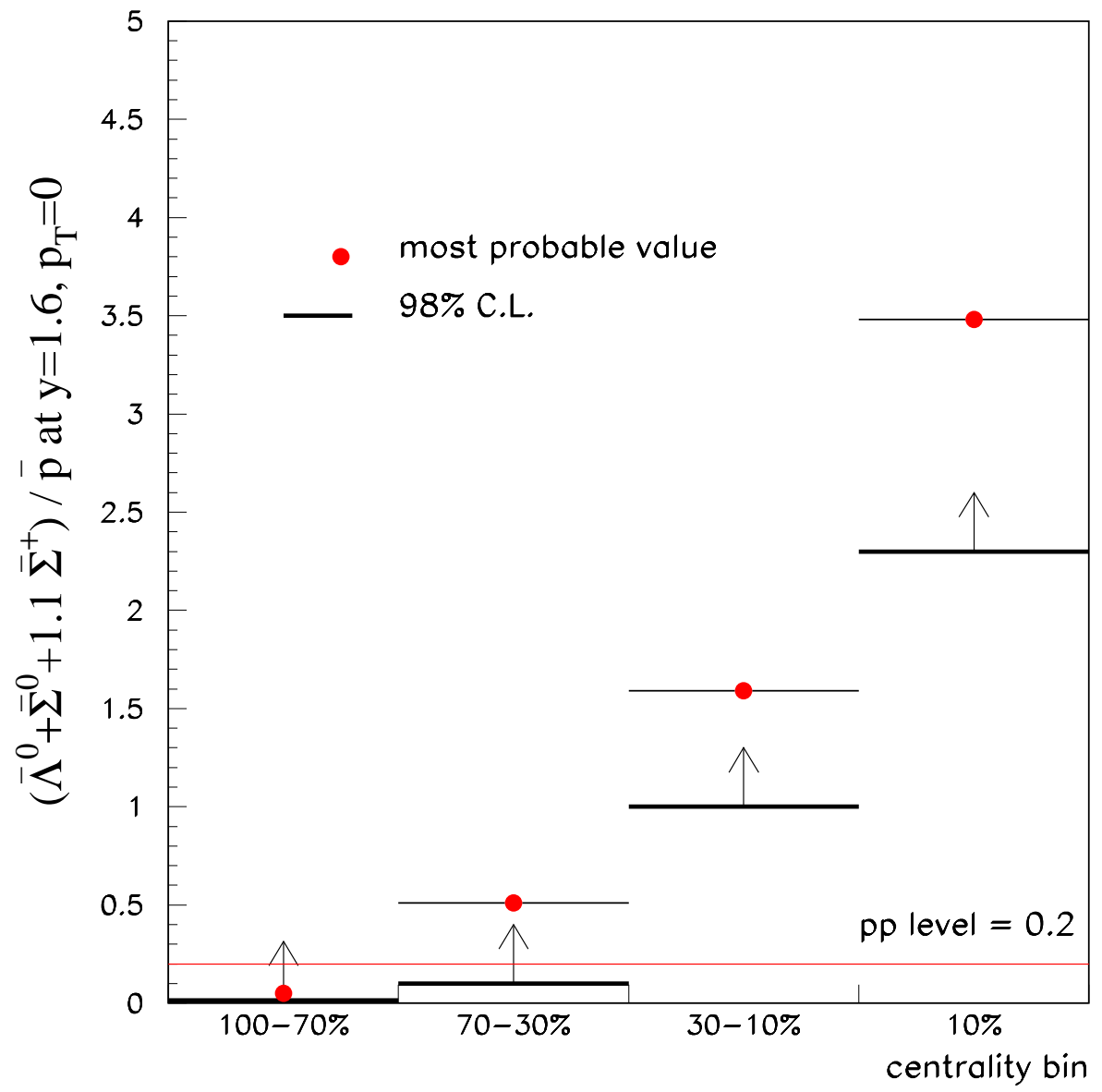

FIG. 12. 98\% confidence level values for the antihyperon/antiproton as a function of centrality. The nominal pp level at $12 \mathrm{GeV} / \mathrm{c}$ is marked. Note the dramatic rise in the ratio as a function of collision centrality. 


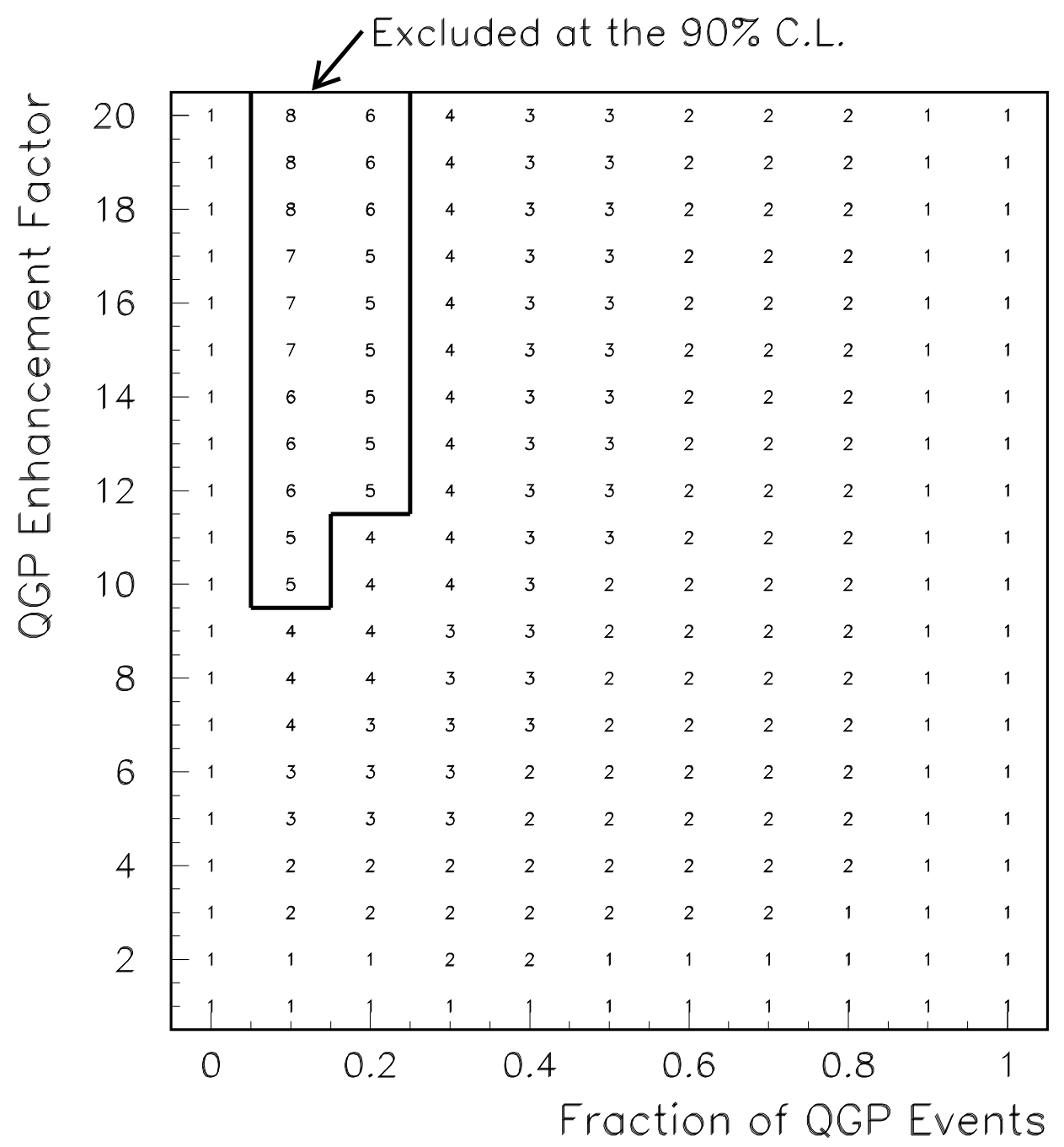

FIG. 13. The number of expected double antiproton events as a function of the fraction of total events which have a QGP formation and the relative antimatter enhancement factor in these QGP events to normal hadronic events. 


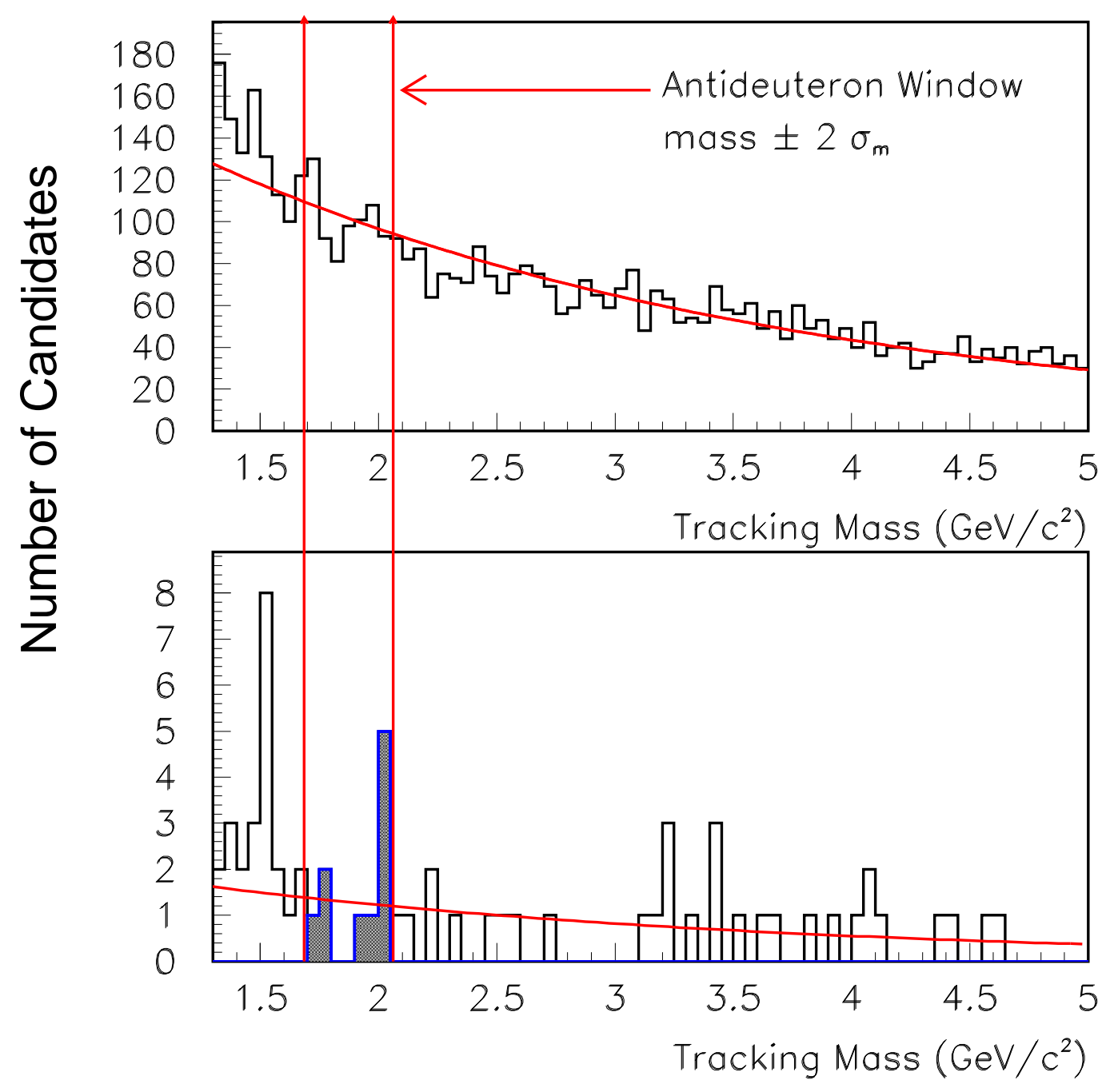

FIG. 14. The upper panel shows the antideuteron candidate tracking mass distribution with no requirement on the calorimeter mass. The bottom panels shown the same distribution after a cut requiring the mass determined from the calorimeter cluster be larger than $1.6 \mathrm{GeV} / c^{2}$. The vertical lines show the antideuteron mass window used in the analysis. 


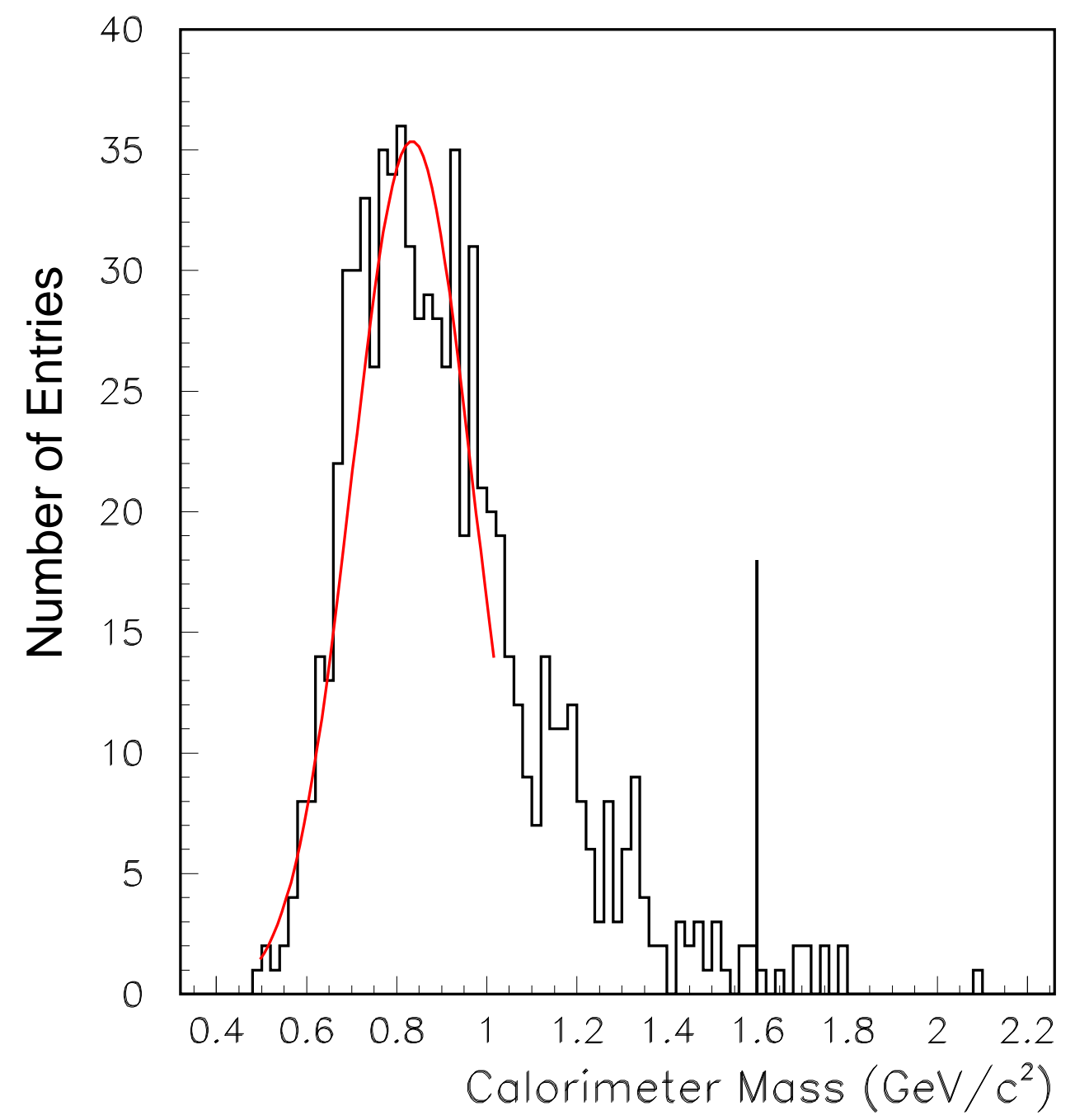

FIG. 15. Calorimeter reconstructed mass for antideuteron candidates with tracking mass $1.687<m<2.061 \mathrm{GeV} / \mathrm{c}^{2}$. The line shows the mass cut at $1.6 \mathrm{GeV} / \mathrm{c}^{2}$ applied in the analysis. 


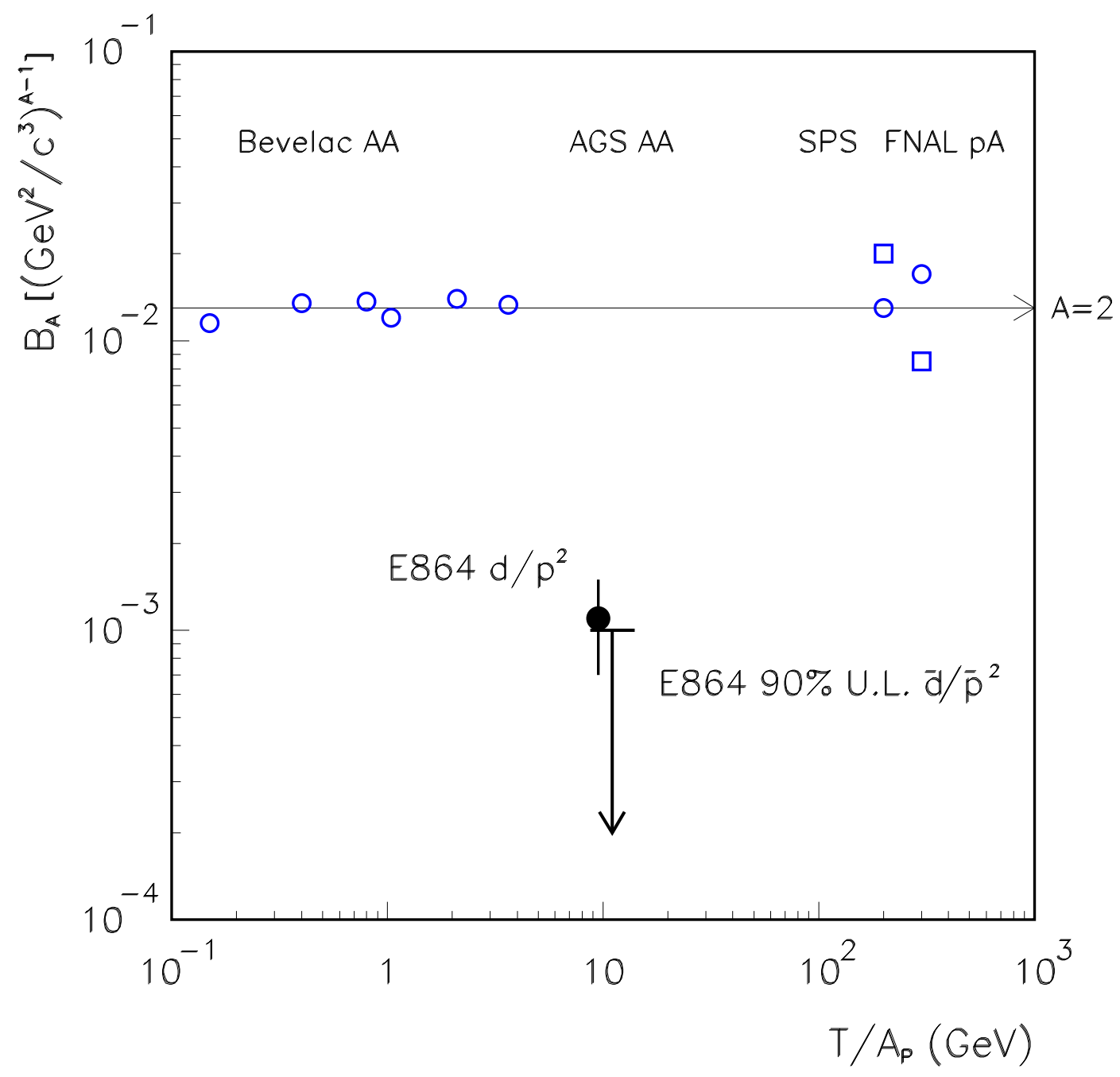

FIG. 16. Coalescence scale factors as a function of kinetic energy per nucleon of the colliding beam. The upper limit on $\overline{B_{2}}$ from E864 is shown as an arrow. The E864 value for deuterons $B_{2}$ is also shown. The simple coalescence level is shown as a line with low energy AA and high energy pA results. 